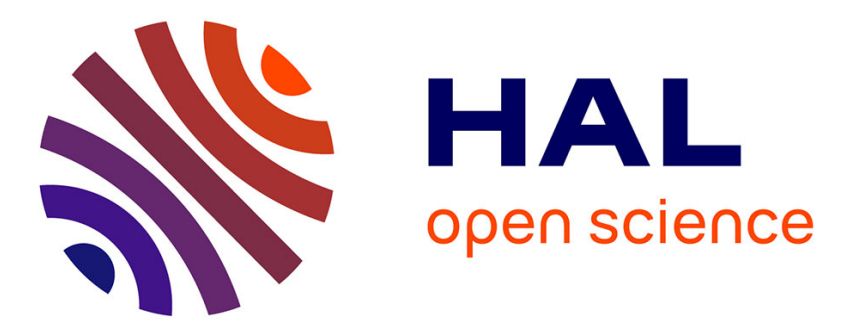

\title{
Galerkin finite element methods with symmetric pressure stabilization for the transient Stokes' equations: stability and convergence analysis
}

Erik Burman, Miguel Angel Fernández

\section{- To cite this version:}

Erik Burman, Miguel Angel Fernández. Galerkin finite element methods with symmetric pressure stabilization for the transient Stokes' equations: stability and convergence analysis. SIAM Journal on Numerical Analysis, 2008, 47 (1), pp.409-439. 10.1137/070707403 . inria-00178359v3

HAL Id: inria-00178359

https://hal.inria.fr/inria-00178359v3

Submitted on 24 Jun 2008

HAL is a multi-disciplinary open access archive for the deposit and dissemination of scientific research documents, whether they are published or not. The documents may come from teaching and research institutions in France or abroad, or from public or private research centers.
L'archive ouverte pluridisciplinaire HAL, est destinée au dépôt et à la diffusion de documents scientifiques de niveau recherche, publiés ou non, émanant des établissements d'enseignement et de recherche français ou étrangers, des laboratoires publics ou privés. 


\section{N R I A}

INSTITUT NATIONAL DE RECHERCHE EN INFORMATIQUE ET EN AUTOMATIQUE

Galerkin finite element methods with symmetric pressure stabilization for the transient Stokes' equations: stability and convergence analysis

Erik Burman — Miguel A. Fernández

$\mathbf{N}^{\circ} 6321$

October 2007

Thème BIO

apport

de recherche 



\title{
RINRIA
}

\section{Galerkin finite element methods with symmetric pressure stabilization for the transient Stokes' equations: stability and convergence analysis}

\author{
Erik Burman*, Miguel A. Fernándeð† \\ Thème BIO - Systèmes biologiques \\ Projet REO \\ Rapport de recherche $\mathrm{n}^{\circ} 6321$ - October 2007 - 35 pages
}

\begin{abstract}
We consider the stability and convergence analysis of pressure stabilized finite element approximations of the transient Stokes' equation. The analysis is valid for a class of symmetric pressure stabilization operators, but also for standard, inf-sup stable, velocity/pressure spaces without stabilization. Provided the initial data is chosen as a specific (method dependent) Ritz-projection, we get unconditional stability and optimal convergence for both pressure and velocity approximations, in natural norms. For arbitrary interpolations of the initial data, a condition between the space and time discretization parameters has to be verified in order to guarantee pressure stability.
\end{abstract}

Key-words: Transient Stokes' equations, finite element methods, symmetric pressure stabilization, time discretization, Ritz-projection

* University of Sussex, UK; e-mail: E.N.Burman@sussex.ac.uk

${ }^{\dagger}$ INRIA, REO team; e-mail: miguel.fernandez@inria.fr 


\section{Méthodes de Galerkin avec stabilisation symétrique de la pression pour l'équation de Stokes transitoire: analyse de stabilité et de convergence}

Résumé : On considère l'analyse de stabilité et convergence de méthodes d'éléments finis stabilisées pour l'équation de Stokes transitoire. L'analyse est valable pour des stabilisations symétriques et faiblements consistantes de la pression. Si l'approximation de la vitesse initiale est donnée par un pro jection de Ritz spécifique, dépendante de la stabilisation, on montre stabilité inconditionnelle et convergence optimale pour la vitesse et la pression, dans des normes naturelles. Par contre, pour des approximations arbitraires de la vitesse initiale, la stabilité de la pression est soumise à une condition entre les param'etres de discrétisation en espace et en temps.

Mots-clés : Équation de Stokes transitoire, méthode d'éléments finis, stabilisation symétrique de la pression, discrétisation en temps, projection de Ritz 


\section{Introduction}

In this paper we consider stabilized finite element methods for the transient Stokes' problem. For standard pressure stabilizations of Petrov-Galerkin (PSPG) or least squares (GaLS) type, the analysis of time-discretization schemes is a difficult issue, unless a space-time approach is applied with a discontinuous Galerkin discretization in time. Indeed, for standard finite difference type time discretizations, the finite difference term must be included in the stabilization operator to assure consistency (see e.g. [11, 23]). It has been shown, in [3, that even for first order backward difference schemes (where the consistency does not improve convergence) this perturbs the stability of the numerical scheme when the time step is small, unless a condition between the space mesh size and the time step is verified,

$$
\delta t \geq C h^{2},
$$

where $\delta t$ denotes the time-step and $h$ the space discretization parameter. For higher order schemes, such as Crank-Nicholson or second order backward differencing, the strongly consistent scheme appears to be unstable (see e.g [1]). Similar initial time-step instabilities were observed, in [19, for the algebraic (static) sub-scale stabilization scheme applied to the Navier-Stokes' equations, and they were cured by including time dependent sub-scales.

Our aim in this work is to consider a fairly large class of pressure stabilization methods and show that convergence of velocities and pressures, for the transient Stokes' problem, can be obtained without conditions on the space and time discretization parameters (like (1)), provided the initial data is chosen as a specific (method dependent) Ritz-projection (see e.g. 33, 34) onto a space of discretely divergence free functions. Discretely divergence free should here be interpreted in the sense of the stabilized method. If, on the other hand, the initial data is chosen as some interpolant that does not conserve the discrete divergence free character, the following condition

$$
\delta t \geq \tilde{C} h^{2 k},
$$

with $k$ the polynomial degree of the velocity approximation space, has to be respected in order to avoid pressure oscillations in the transient solution for small times.

Although the stability conditions (1) and (2) are similar, their nature is different. As mentioned above, if (2) fails to be satisfied pressure instabilities appear when dealing with non-discrete divergence free initial velocity approximation, but they are not related to the structure of the pressure stabilization. For residual based stabilization methods (PSPG, GaLS, etc.), on the other hand, the finite difference/pressure coupling of the stabilization perturbs the coercivity of the discrete pressure operator (see [3]) unless condition (1) is satisfied (irrespectively of the divergence free character of the initial velocity approximation).

The analysis carried out in this paper is valid for pressure stabilization operators that are symmetric and weakly consistent, as well as for standard methods using inf-sup stable velocity/pressure pairs, but it does not apply to residual based pressure stabilizations (PSPG, GaLS, etc.). In particular, space and time discretization commute (i.e. lead to the same fully discrete scheme) for the methods we analyze. 
We prove unconditional stability of velocities and pressures and optimal convergence (in natural norms) when the initial data is chosen as a certain Ritz-type projection. In case a standard interpolation of the initial data is applied, an inverse parabolic CFL-type condition must be respected in order to maintain pressure stability for small time-steps. We give the full analysis only for the backward difference formula of order one, and we indicate how the analysis changes in the case of second order approximations in time. Indeed any $\mathcal{A}$-stable implicit scheme is expected to yield optimal performance.

The remainder of the paper is organized as follows. In the next section we introduce the problem under consideration and some useful notation. The space and time discretized formulations are introduced in section $\$ 3$. In paragraph $\$ 3.1$ the space discretization is formulated using a general framework, we also discuss how some known pressure stabilized finite element methods enter this setting. The time discretization is performed in paragraph 33.2 using the BDF1, Crank-Nicholson or BDF2 schemes. Section $\$ 4$ is devoted to the stability analysis of the resulting fully discrete formulations. The convergence analysis for the BDF1 scheme is carried out in section \$5. We illustrate the theoretical results with some numerical experiments in section $\$ 6$, using interior penalty stabilization of the gradient jumps. Finally, some conclusions are given in section 87

\section{Problem setting}

Let $\Omega$ be a domain in $\mathbb{R}^{d}(d=2$ or 3$)$ with a polyhedral boundary $\partial \Omega$. For $T>0$ we consider the problem of solving, for $\boldsymbol{u}: \Omega \times(0, T) \longrightarrow \mathbb{R}^{d}$ and $p: \Omega \times(0, T) \longrightarrow \mathbb{R}$, the following time-dependent Stokes problem:

$$
\left\{\begin{aligned}
\partial_{t} \boldsymbol{u}-\nu \Delta \boldsymbol{u}+\nabla p=\boldsymbol{f}, & \text { in } \Omega \times(0, T), \\
\boldsymbol{\nabla} \cdot \boldsymbol{u}=0, & \text { in } \Omega \times(0, T), \\
\boldsymbol{u}=\mathbf{0}, & \text { on } \partial \Omega \times(0, T), \\
\boldsymbol{u}(\cdot, 0)=\boldsymbol{u}_{0}, & \text { in } \Omega .
\end{aligned}\right.
$$

Here, $\boldsymbol{f}: \Omega \times(0, T) \longrightarrow \mathbb{R}$ stands for the source term, $\boldsymbol{u}_{0}: \Omega \longrightarrow \mathbb{R}^{d}$ for the initial velocity and $\nu>0$ for a given constant viscosity. In order to introduce a variational setting for (3) we consider the following standard velocity and pressure spaces

$$
V \stackrel{\text { def }}{=}\left[H_{0}^{1}(\Omega)\right]^{d}, \quad H \stackrel{\text { def }}{=}\left[L^{2}(\Omega)\right]^{d}, \quad Q \stackrel{\text { def }}{=} L_{0}^{2}(\Omega),
$$

normed with

$$
\|\boldsymbol{v}\|_{H} \stackrel{\text { def }}{=}(\boldsymbol{v}, \boldsymbol{v})^{\frac{1}{2}}, \quad\|\boldsymbol{v}\|_{V} \stackrel{\text { def }}{=}\left\|\nu^{\frac{1}{2}} \nabla \boldsymbol{v}\right\|_{H}, \quad\|q\|_{Q} \stackrel{\text { def }}{=}\left\|\nu^{-\frac{1}{2}} q\right\|_{H},
$$

where $(\cdot, \cdot)$ denotes the standard $L^{2}$-inner product in $\Omega$.

Problem (3) can be formulated in weak form as follows: For all $t>0$, find $\boldsymbol{u}(t) \in V$ and $p(t) \in Q$ such that

$$
\left\{\begin{aligned}
\left(\partial_{t} \boldsymbol{u}, \boldsymbol{v}\right)+a(\boldsymbol{u}, \boldsymbol{v})+b(p, \boldsymbol{v})=(\boldsymbol{f}, \boldsymbol{v}), & \text { a.e. in } \quad(0, T), \\
b(q, \boldsymbol{u})=0, & \text { a.e. in }(0, T), \\
\boldsymbol{u}(\cdot, 0)=\boldsymbol{u}_{0}, & \text { a.e. in } \Omega,
\end{aligned}\right.
$$


for all $\boldsymbol{v} \in V, q \in Q$ and with

$$
a(\boldsymbol{u}, \boldsymbol{v}) \stackrel{\text { def }}{=}(\nu \boldsymbol{\nabla} \boldsymbol{u}, \boldsymbol{\nabla} \boldsymbol{v}), \quad b(p, \boldsymbol{v}) \stackrel{\text { def }}{=}-(p, \boldsymbol{\nabla} \cdot \boldsymbol{v}) .
$$

From these definitions, the following classical coercivity and continuity estimates hold:

$$
a(\boldsymbol{v}, \boldsymbol{v}) \geq\|\boldsymbol{v}\|_{V}^{2}, \quad a(\boldsymbol{u}, \boldsymbol{v}) \leq\|\boldsymbol{u}\|_{V}\|\boldsymbol{v}\|_{V}, \quad b(\boldsymbol{v}, q) \leq\|\boldsymbol{v}\|_{V}\|q\|_{Q},
$$

for all $\boldsymbol{u}, \boldsymbol{v} \in V$ and $q \in Q$. It is known (see e.g. [22]) that if $\boldsymbol{f} \in C^{0}([0, T] ; H)$ and that $\boldsymbol{u}_{0} \in V \cap H_{0}(\operatorname{div} ; \Omega)$ problem (4) admits a unique solution $(\boldsymbol{u}, p)$ in $L^{2}(0, T ; V) \times L^{2}(0, T ; Q)$ with $\partial_{t} \boldsymbol{u} \in L^{2}\left(0, T ; V^{\prime}\right)$.

Thorough this paper, $C$ stands for a generic positive constant independent of the physical and discretization parameters.

\section{Space and time discretization}

In this section we discretize problem (4) with respect to the space and time variables. Symmetric pressure stabilized finite elements are used for the space discretization (paragraph $\$ 3.1$, and some known $\mathcal{A}$-stable schemes for the time discretization (paragraph 3.2 .

\subsection{Space semi-discretization: symmetric pressure stabi- lized formulations}

Let $\left\{\mathcal{T}_{h}\right\}_{0<h \leq 1}$ denote a shape-regular family of triangulations of the domain $\Omega$. For each triangulation $\mathcal{T}_{h}$, the subscript $h \in(0,1]$ refers to the level of refinement of the triangulation, which is defined by

$$
h \stackrel{\text { def }}{=} \max _{K \in \mathcal{T}_{h}} h_{K}
$$

with $h_{K}$ the diameter of $K$. In order to simplify the analysis we assume that the family of triangulations $\left\{\mathcal{T}_{h}\right\}_{0<h \leq 1}$ is quasi-uniform. For more precise information on the constraint on the mesh we refer to the analysis of the various finite element methods in the steady case, see paragraph 33.1 .1

In this paper, we let $X_{h}^{k}$ and $M_{h}^{l}$ denote, respectively, the standard spaces of continuous and (possibly) discontinuous piecewise polynomial functions of degree $k \geq 1$ and $l \geq 0(k-1 \leq l \leq k)$,

$$
\begin{aligned}
& X_{h}^{k} \stackrel{\text { def }}{=}\left\{v_{h} \in C^{0}(\bar{\Omega}): v_{h \mid K} \in \mathbb{P}_{k}(K), \quad \forall K \in \mathcal{T}_{h}\right\}, \\
& M_{h}^{l} \stackrel{\text { def }}{=}\left\{q_{h} \in L^{2}(\Omega): q_{h \mid K} \in \mathbb{P}_{l}(K), \quad \forall K \in \mathcal{T}_{h}\right\} .
\end{aligned}
$$

For the approximated velocities we will use the space $\left[V_{h}^{k}\right]^{d} \stackrel{\text { def }}{=}\left[X_{h}^{k} \cap H_{0}^{1}(\Omega)\right]^{d}$ and for the pressure we will use either $Q_{h}^{l} \stackrel{\text { def }}{=} M_{h}^{l} \cap L_{0}^{2}(\Omega)$ or $Q_{h}^{l} \stackrel{\text { def }}{=} M_{h}^{l} \cap$ $L_{0}^{2}(\Omega) \cap C^{0}(\bar{\Omega})$. In order to stabilize the pressure we introduce a bi-linear form $j: Q_{h} \times Q_{h} \longrightarrow \mathbb{R}$ satisfying the following properties:

- Symmetry:

$$
j\left(p_{h}, q_{h}\right)=j\left(q_{h}, p_{h}\right), \quad \forall p_{h}, q_{h} \in Q_{h}^{l},
$$


- Continuity:

$$
\left|j\left(p_{h}, q_{h}\right)\right| \leq j\left(p_{h}, p_{h}\right)^{\frac{1}{2}} j\left(q_{h}, q_{h}\right)^{\frac{1}{2}} \leq C\left\|p_{h}\right\|_{Q}\left\|q_{h}\right\|_{Q}, \quad \forall p_{h}, q_{h} \in Q_{h}^{l},
$$

- Weak consistency:

$$
j\left(\Pi_{h}^{l} q, \Pi_{h}^{l} q\right)^{\frac{1}{2}} \leq C \frac{h^{s_{p}}}{\nu}\|q\|_{s_{p}, \Omega}, \quad \forall q \in H^{s}(\Omega),
$$

with $s_{p} \stackrel{\text { def }}{=} \min \{s, \tilde{l}, l+1\}, \tilde{l} \geq 1$ denoting the order of weak consistency of the stabilization operator, and $\Pi_{h}^{l}: Q \longrightarrow Q_{h}^{l}$ a given projection operator such that

$$
\left\|q-\Pi_{h}^{l} q\right\|_{Q} \leq \frac{C}{\nu^{\frac{1}{2}}} h^{l+1}\|q\|_{l+1, \Omega}
$$

for all $q \in H^{l+1}(\Omega)$.

Finally, we assume that there exists a projection operator $\mathcal{I}_{h}^{k}: V \longrightarrow V_{h}^{k}$ satisfying the following approximation properties:

$$
\begin{aligned}
& \left\|\boldsymbol{v}-\mathcal{I}_{h}^{k} \boldsymbol{v}\right\|_{H}+h \nu^{-\frac{1}{2}}\left\|\boldsymbol{v}-\mathcal{I}_{h}^{k} \boldsymbol{v}\right\|_{V} \leq C_{\mathcal{I}} h^{r_{u}}\|\boldsymbol{v}\|_{r_{u}, \Omega}, \\
& \left|b\left(q_{h}, \boldsymbol{v}-\mathcal{I}_{h}^{k} \boldsymbol{v}\right)\right| \leq C j\left(q_{h}, q_{h}\right)^{\frac{1}{2}}\left(\nu^{\frac{1}{2}}\left\|h^{-1}\left(\boldsymbol{v}-\mathcal{I}_{h}^{k} \boldsymbol{v}\right)\right\|_{H}+\left\|\boldsymbol{v}-\mathcal{I}_{h}^{k} \boldsymbol{v}\right\|_{V}\right),
\end{aligned}
$$

for all $\boldsymbol{v} \in\left[H^{r}(\Omega)\right]^{d}, r_{\boldsymbol{u}}=\min \{r, k+1\}$ and $\left(q_{h}, \boldsymbol{v}_{h}\right) \in Q_{h}^{l} \times\left[V_{h}^{k}\right]^{d}$.

Our space semi-discretized scheme reads: For all $t \in(0, T)$, find $\left(\boldsymbol{u}_{h}(t), p_{h}(t)\right) \in$ $\left[V_{h}^{k}\right]^{d} \times Q_{h}^{l}$ such that

$$
\begin{aligned}
\left(\partial_{t} \boldsymbol{u}_{h}, \boldsymbol{v}_{h}\right)+a\left(\boldsymbol{u}_{h}, \boldsymbol{v}_{h}\right)+b\left(p_{h}, \boldsymbol{v}_{h}\right)-b\left(q_{h}, \boldsymbol{u}_{h}\right)+j\left(p_{h}, q_{h}\right) & =\left(\boldsymbol{f}, \boldsymbol{v}_{h}\right), \\
\boldsymbol{u}_{h}(0) & =\boldsymbol{u}_{h}^{0},
\end{aligned}
$$

for all $\left(\boldsymbol{v}_{h}, q_{h}\right) \in\left[V_{h}^{k}\right]^{d} \times Q_{h}^{l}$ and with $\boldsymbol{u}_{h}^{0}$ a suitable approximation of $\boldsymbol{u}_{0}$ in $\left[V_{h}^{k}\right]^{d}$.

The following modified inf-sup condition states the stability of the discrete pressures in 12.

Lemma 3.1 There exists two constants $C, \beta>0$, independent of $h$ and $\nu$, such that

$$
\sup _{\boldsymbol{v}_{h} \in\left[V_{h}^{k}\right] d} \frac{\left|b\left(q_{h}, \boldsymbol{v}_{h}\right)\right|}{\left\|\boldsymbol{v}_{h}\right\|_{V}}+C j\left(q_{h}, q_{h}\right)^{\frac{1}{2}} \geq \beta\left\|q_{h}\right\|_{Q},
$$

for all $q_{h} \in Q_{h}^{l}$.

Proof. Let $q_{h} \in Q_{h}^{l}$, from [25, Corollary 2.4] and (10) there exists $\boldsymbol{v}_{q} \in H_{0}^{1}(\Omega)$ such that $\boldsymbol{\nabla} \cdot \boldsymbol{v}_{q}=\nu^{-1} q_{h}$ and

$$
\left\|\mathcal{I}_{h}^{k} \boldsymbol{v}_{q}\right\|_{V} \leq C\left\|\boldsymbol{v}_{q}\right\|_{V} \leq C\left\|q_{h}\right\|_{Q}
$$

On the other hand, using [11, we have

$$
\begin{aligned}
\left\|q_{h}\right\|_{Q}^{2} & =b\left(q_{h}, \boldsymbol{v}_{q}\right) \\
& =b\left(q_{h}, \boldsymbol{v}_{q}-\mathcal{I}_{h}^{k} \boldsymbol{v}_{q}\right)+b\left(q_{h}, \mathcal{I}_{h}^{k} \boldsymbol{v}_{q}\right) \\
& \leq C j\left(q_{h}, q_{h}\right)^{\frac{1}{2}}\left(\left\|\nu^{\frac{1}{2}} h^{-1}\left(\boldsymbol{v}-\mathcal{I}_{h}^{k} \boldsymbol{v}\right)\right\|_{H}+\left\|\boldsymbol{v}-\mathcal{I}_{h}^{k} \boldsymbol{v}\right\|_{V}\right)+b\left(q_{h}, \mathcal{I}_{h}^{k} \boldsymbol{v}_{q}\right) \\
& \leq C j\left(q_{h}, q_{h}\right)^{\frac{1}{2}}\left\|q_{h}\right\|_{Q}+b\left(q_{h}, \mathcal{I}_{h}^{k} \boldsymbol{v}_{q}\right) .
\end{aligned}
$$


We conclude the proof by dividing this last inequality by $\left\|\mathcal{I}_{h}^{k} \boldsymbol{v}_{q}\right\|_{V}$ and using (14).

The above Lemma assures the well-posedness of problem (12). This is stated in the following Theorem.

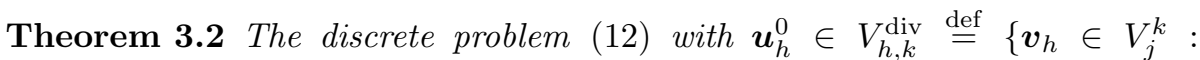
$\left.b\left(q_{h}, \boldsymbol{v}_{h}\right)=0, \quad \forall q_{h} \in Q_{h} \cap \operatorname{Ker} j\right\}$ has a unique solution $\left(\boldsymbol{u}_{h}, p_{h}\right) \in C^{1}\left((0, T] ;\left[V_{h}^{k}\right]^{d}\right) \times$ $C^{0}\left((0, T] ; Q_{h}^{k}\right)$.

To facilitate the analysis we introduce the following (mesh-dependent) seminorm, which is a norm for the velocity and a semi-norm for the pressure,

$$
\left\|\left(\boldsymbol{v}_{h}, q_{h}\right)\right\|_{h}^{2} \stackrel{\text { def }}{=}\left\|\boldsymbol{v}_{h}\right\|_{V}^{2}+j\left(q_{h}, q_{h}\right) .
$$

Remark 3.3 If the velocity/pressure finite-element pair $V_{h}^{k} / Q_{h}^{k}$ is inf-sup stable, we can take $j(\cdot, \cdot) \stackrel{\text { def }}{=} 0$ in $(12)$, as usual. Obviously, this choice is compatible with hypothesis (6)-(8) so that the results of this paper still apply. In particular, the relation 13 becomes the standard inf-sup condition between $V_{h}^{k}$ and $Q_{h}^{k}$.

\subsubsection{Examples}

In this section we will review some of the most well-known pressure projection stabilization methods and discuss how they enter the abstract framework of the previous paragraph. For detailed results on analysis for the respective methods we refer the reader to the references considering the stationary case.

Recently, several different weakly consistent symmetric pressure stabilized finite element methods have been proposed. These methods take their origin in the work of Silvester [32] and the work by Codina and Blasco [17. Further developments include the work by Becker and Braack [2] on local projection schemes, the extension of the interior penalty method, using penalization of gradient jumps, to the case of pressure stabilization by Burman and Hansbo [14 and the interpretation of these methods as minimal stabilization procedures by Brezzi and Fortin [9]. Similar approaches have been advocated in Bochev and Dohrmann in 21] and a review of the analysis (with special focus on discontinuous pressure spaces and the Darcy problem) is given in [12].

The main idea underpinning all these methods is that, when using a velocitypressure space pair $V_{h} \times Q_{h}$, the inf-sup stability constraint on the spaces may be relaxed by the addition of an operator penalizing the difference between the discrete pressure variable and its projection onto a subspace $\tilde{Q}_{h} \subset Q_{h}$, such that $V_{h} \times \tilde{Q}_{h}$ is inf-sup stable. The penalization may either act directly on the pressure, as in 21, 12], or on the gradient of the pressure, as in 2, 18, 14. Generally speaking, the pressure approximation properties of the numerical scheme will be given by $\tilde{Q}_{h}$, expressed in the weak consistency satisfied by the penalty operator. In some cases [13, 6, 16, these stabilized methods may be extended to include high Reynolds number effects in the case of the Oseen's problem. The advantages and disadvantages of symmetric weakly consistent pressure stabilization methods compared to GaLS or PSPG approaches is discussed in a recent review paper $\mathbf{7}$. 
The methods of Brezzi-Pitkäranta, Silvester and Bochev-Dohrmann

The original pressure stabilized finite element method was proposed by Brezzi and Pitkäranta in [10. Here, the velocity and pressure discrete spaces are chosen as the standard finite element space of piecewise affine continuous functions, $\left[V_{h}^{1}\right]^{d} \times Q_{h}^{1}$. The operator $j(\cdot, \cdot)$ is given by

$$
j\left(p_{h}, q_{h}\right)=\left(\frac{h^{2}}{\nu} \nabla p_{h}, \nabla q_{h}\right) .
$$

A variant of this method was recently proposed by Dohrmann and Bochev, in 21], using an equivalent stabilization operator, namely,

$$
j\left(p_{h}, q_{h}\right)=\left(\frac{1}{\nu}\left(I-\pi_{0}\right) p_{h},\left(I-\pi_{0}\right) q_{h}\right),
$$

where $\pi_{0}: Q \longrightarrow Q_{h}^{0}$ denotes the (element-wise) projection onto piecewise constants. Property (11) is verified after an integration by parts, with $\mathcal{I}_{h}^{1}$ simply the Scott-Zhang interpolant onto $\left[V_{h}^{1}\right]^{d}$ (see e.g. [31, 22])

$$
\begin{aligned}
b\left(q_{h}, \boldsymbol{v}-\mathcal{I}_{h}^{1} \boldsymbol{v}\right) & =\left(\boldsymbol{\nabla} q_{h}, \boldsymbol{v}-\mathcal{I}_{h}^{1} \boldsymbol{v}\right) \\
& \leq j\left(q_{h}, q_{h}\right)^{\frac{1}{2}}\left(h^{-1} \nu^{\frac{1}{2}}\left\|\boldsymbol{v}-\mathcal{I}_{h}^{1} \boldsymbol{v}\right\|_{H}+\left\|\boldsymbol{v}-\mathcal{I}_{h}^{1} \boldsymbol{v}\right\|_{V}\right) .
\end{aligned}
$$

One readily verifies that (7) and (8) hold. Moreover, in both cases, (16) and (17), the weak consistency property holds (with $\tilde{l}=1$ )

$$
j\left(\Pi_{h}^{1} p, \Pi_{h}^{1} p\right)^{\frac{1}{2}} \leq \frac{C}{\nu^{\frac{1}{2}}} h\|p\|_{1, \Omega},
$$

with $\Pi_{h}^{1}$ being, for instance, the $L^{2}$-projection onto $Q_{h}^{1}$ (we could use instead the Clément [15 or Scott-Zhang interpolants). Indeed, for (16) we apply the $H^{1}$-stability of the $L^{2}$-projection (see e.g. $\left.22,20,8,5\right]$ ), whereas for (17) we add and subtract suitable terms $\left(p\right.$ and $\left.\pi_{0} p\right)$ and use the approximation properties of $\pi_{0}$ and $\Pi_{h}^{1}$ (see e.g. [22]). As a result, our analysis for the time discretization is valid.

Another low order scheme, covered by the analysis, is the method which consists in using piecewise affine continuous velocities and element wise constants pressures, $\left[V_{h}^{1}\right]^{d} \times Q_{h}^{0}$, see e.g. $[27$. Stability is obtained by the addition of the jump over element faces of the discontinuous pressure, namely,

$$
j\left(p_{h}, q_{h}\right)=\sum_{K \in \mathcal{T}_{h}} \int_{\partial K \backslash \partial \Omega} \frac{h}{\nu} \llbracket p_{h} \rrbracket \llbracket q_{h} \rrbracket .
$$

Here, $\llbracket q_{h} \rrbracket$ denotes the jump of $q_{h}$ over the inter-element boundary, defined by,

$$
\llbracket q_{h} \rrbracket(\boldsymbol{x}) \stackrel{\text { def }}{=} \lim _{\epsilon \rightarrow 0}\left(q_{h}(\boldsymbol{x}+\epsilon \boldsymbol{n})-q_{h}(\boldsymbol{x}-\epsilon \boldsymbol{n})\right), \quad \forall \boldsymbol{x} \in F,
$$

with $\boldsymbol{n}$ standing for a fixed, but arbitrary, normal to the internal face $F$. In this case, (11) is obtained after an integration by parts in the term $b\left(q_{h}, \boldsymbol{v}-\mathcal{I}_{h}^{1} \boldsymbol{v}\right)$ and an element-wise trace inequality,

$$
\begin{aligned}
b\left(q_{h}, \boldsymbol{v}-\mathcal{I}_{h}^{1} \boldsymbol{v}\right) & =-\sum_{K} \int_{\partial K \backslash \partial \Omega} \llbracket q_{h} \rrbracket\left(\boldsymbol{v}-\mathcal{I}_{h}^{1} \boldsymbol{v}\right) \cdot \boldsymbol{n} \\
& \leq j\left(q_{h}, q_{h}\right)^{\frac{1}{2}}\left(h^{-1} \nu^{\frac{1}{2}}\left\|\boldsymbol{v}-\mathcal{I}_{h}^{1} \boldsymbol{v}\right\|_{H}+\left\|\boldsymbol{v}-\mathcal{I}_{h}^{1} \boldsymbol{v}\right\|_{V}\right) .
\end{aligned}
$$


In addition, by taking, for instance, $\Pi_{h}^{0}$ as the $L^{2}$-projection onto $Q_{h}^{0}$, using an element-wise trace inequality and the approximation properties of $\Pi_{h}^{0}$ (see e.g. [22]), one also easily shows that the weak consistency property holds,

$$
j\left(\Pi_{h}^{0} p, \Pi_{h}^{0} p\right)^{\frac{1}{2}}=j\left(\left(I-\Pi_{h}^{0}\right) p,\left(I-\Pi_{h}^{0}\right) p\right)^{\frac{1}{2}} \leq \frac{C}{\nu^{\frac{1}{2}}} h\|p\|_{1, \Omega},
$$

hence $\tilde{l}=1$.

For details on the cases of stabilization of the pressure jumps only in macro elements, or the generalization to higher order finite element spaces of the Taylor-Hood family with discontinuous pressures, we refer to [12].

\section{Orthogonal sub-scale stabilization}

The orthogonal sub-scale stabilization was proposed by Codina and Blasco in [17. Equal order $(k=l \geq 1)$ continuous approximation spaces are used for the velocities and the pressures.

Here the main idea is to penalize the difference between the pressure gradient and its projection onto the finite element space. This imposes the introduction of an auxiliary variable for the projection since it may not be localized and is given only implicitly. Hence, the stabilization operator is given by

$$
j\left(p_{h}, q_{h}\right)=\left(\frac{h^{2}}{\nu}\left(\nabla p_{h}-\pi_{h}^{k} \nabla p_{h}\right), \nabla q_{h}\right),
$$

where $\pi_{h}^{k}:\left[L^{2}(\Omega)\right]^{d} \longrightarrow\left[V_{h}^{k}\right]^{d}$ stands for the $L^{2}$-projection onto $\left[V_{h}^{k}\right]^{d}$, which is given as the solution of the (global) problem

$$
\left(\pi_{h}^{k} \nabla p_{h}, \boldsymbol{\xi}_{h}\right)=\left(\nabla p_{h}, \boldsymbol{\xi}_{h}\right), \quad \forall \boldsymbol{\xi}_{h} \in\left[V_{h}^{k}\right]^{d} .
$$

One may readily show that (7) and (8) hold. Disregarding for simplicity the boundary conditions, the projection operator $\mathcal{I}_{h}^{k}=\pi_{h}^{k}$ of (11) is here chosen also as the $L^{2}$-projection onto $\left[V_{h}^{k}\right]^{d}$. This can be justified if boundary conditions are imposed weakly, for instance using Nitsche's method (see [29, 24]), and $V_{h}^{k}$ includes the degrees of freedom on the boundary. Indeed, then we have

$$
\begin{aligned}
b\left(q_{h}, \boldsymbol{v}-\mathcal{I}_{h}^{k} \boldsymbol{v}\right) & =\left(\boldsymbol{\nabla} q_{h}-\pi_{h}^{k} \boldsymbol{\nabla} q_{h}, \boldsymbol{v}-\mathcal{I}_{h}^{k} \boldsymbol{v}\right) \\
& \leq j\left(q_{h}, q_{h}\right)^{\frac{1}{2}}\left(h^{-1} \nu^{\frac{1}{2}}\left\|\boldsymbol{v}-\mathcal{I}_{h}^{k} \boldsymbol{v}\right\|_{H}+\left\|\boldsymbol{v}-\mathcal{I}_{h}^{k} \boldsymbol{v}\right\|_{V}\right) .
\end{aligned}
$$

Finally, by taking $\Pi_{h}^{k}: Q \longrightarrow Q_{h}^{k}$ as the $L^{2}$-projection onto $Q_{h}^{k}$, adding and subtracting suitable terms $\left(\nabla p\right.$ and $\left.\pi_{h}^{k} \nabla p\right)$ and using the approximation properties of $\pi_{h}^{k}$ and $\Pi_{h}^{k}$ (see e.g. [22]), one readily verifies the weak consistency

$$
j\left(\Pi_{h}^{k} p, \Pi_{h}^{k} p\right)^{\frac{1}{2}}=\frac{h}{\nu^{\frac{1}{2}}}\left\|\left(I-\pi_{h}^{k}\right) \nabla \Pi_{h}^{k} p\right\|_{0, \Omega} \leq \frac{C}{\nu^{\frac{1}{2}}} h^{s_{p}}\|p\|_{s_{p}, \Omega},
$$

for all $p \in H^{s}(\Omega)$ and with $s_{p}=\min \{k+1, s\}$. In particular, $\tilde{l}=l=k$. The above analysis is hence valid also in this case (with some modifications of technical nature due to the weakly imposed boundary conditions). 


\section{Local projection stabilization}

In the local projection stabilization, proposed in [2], stability is obtained by penalizing the projection of the gradient onto piecewise discontinuous functions defined on patches consisting of several elements, obtained by using hierarchic meshes, or by penalizing the gradient of the difference of the pressure and its projection on polynomials of lower polynomial order. The construction relies on the inf-sup stability of a velocity/pressure pair typically of mini-element character or of the Taylor-Hood family. Similar ideas were advocated in [21]. The stabilization operator is written as

$$
j\left(p_{h}, q_{h}\right)=\sum_{\tilde{K}}\left(\frac{h^{2}}{\nu} \kappa \nabla p_{h}, \kappa \nabla q_{h}\right)
$$

where $\kappa$ is the so called fluctuation operator defined as $\kappa \stackrel{\text { def }}{=} I-\tilde{\pi}_{h}$ where $\tilde{\pi}_{h}$ denotes a local projection operator onto either a polynomial of order $k$ on a macro-patch consisting of three triangles (or four quadrilaterals) or a polynomial of order $k-1$ on the element. One may show that (11), (7) and (8) hold (for details on the construction of $\mathcal{I}_{h}^{k}$ see [2, 6] and for general conditions on the finite element spaces and stabilization operators 28]). In case we consider the projection $\tilde{\pi}_{h}$ onto polynomials of order $k-1$ the stabilization operator may be written as

$$
j\left(p_{h}, q_{h}\right)=\sum_{\tilde{K}}\left(\frac{h^{2}}{\nu} \nabla\left(\kappa p_{h}\right), \nabla\left(\kappa q_{h}\right)\right),
$$

or, equivalently, following [21,

$$
j\left(p_{h}, q_{h}\right)=\sum_{\tilde{K}}\left(\frac{1}{\nu} \kappa p_{h}, \kappa q_{h}\right) .
$$

In these latter cases, the condition 111 is obtained by choosing $\mathcal{I}_{h}^{k}$ as the Fortin interpolation operator associated to $\left[V_{h}^{k}\right]^{d} \times \tilde{Q}_{h}$ where $\tilde{Q}_{h}$ is the space of continuous piecewise polynomial functions of order $k-1$. Clearly we then have

$$
\begin{aligned}
b\left(q_{h}, \boldsymbol{v}-\mathcal{I}_{h}^{k} \boldsymbol{v}\right) & =b\left(\kappa q_{h}, \boldsymbol{v}-\mathcal{I}_{h}^{k} \boldsymbol{v}\right) \\
& \leq j\left(q_{h}, q_{h}\right)^{\frac{1}{2}}\left(h^{-1} \nu^{\frac{1}{2}}\left\|\boldsymbol{v}-\mathcal{I}_{h}^{k} \boldsymbol{v}\right\|_{H}+\left\|\boldsymbol{v}-\mathcal{I}_{h}^{k} \boldsymbol{v}\right\|_{V}\right)
\end{aligned}
$$

since $b\left(\tilde{q}_{h}, \boldsymbol{v}-\mathcal{I}_{h}^{k} \boldsymbol{v}\right)=0$ for all $\tilde{q}_{h} \in \tilde{Q}_{h}$. The form 19 is treated in a similar fashion after an integration by parts. On the other hand, by taking $\Pi_{h}^{l}: Q \longrightarrow$ $Q_{h}^{l}$ as the $L^{2}$-projection operator onto $Q_{h}^{l}$ and using approximation properties of $\Pi_{h}^{l}$ and $\tilde{\pi}_{h}$, we have

$$
\begin{aligned}
j\left(\Pi_{h}^{l} p, \Pi_{h}^{l} p\right)^{\frac{1}{2}} & \leq j\left(\left(I-\Pi_{h}^{l}\right) p,\left(I-\Pi_{h}^{l}\right) p\right)^{\frac{1}{2}}+j(p, p)^{\frac{1}{2}} \\
& \leq \frac{C}{\nu^{\frac{1}{2}}} h^{s_{p}}\|p\|_{s_{p}, \Omega}, \forall p \in H^{s}(\Omega),
\end{aligned}
$$

where $s_{p}=\min \{\tilde{l}, s, l+1\}$ and $\tilde{l}-1$ denotes the polynomial order of the space on which the local projection is taken. Clearly if we project on polynomials of order $k-1$ the stabilization operator looses one order in the weak consistency, however the estimates remain optimal since we expect the velocities to be one order more regular than the pressure. 


\section{Continuous interior penalty stabilization}

The continuous interior penalty stabilization for the stationary Stokes' problem was proposed in [14] and generalized to the Oseen's problem in [13]. It uses equal order continuous approximation spaces for velocities and pressures $(k=l \geq 1)$ and relies on the fact that the component of the pressure gradient orthogonal to the finite element space may be controlled by the gradient jumps using an interpolation estimate between discrete spaces. Indeed it was shown on [13] that the following inequality holds

$$
\left\|h\left(\boldsymbol{\nabla} p_{h}-\tilde{i} \nabla p_{h}\right)\right\|_{H}^{2} \leq \sum_{K \in \mathcal{T}_{h}} \int_{\partial K \backslash \partial \Omega} h_{K}^{3} \llbracket \nabla p_{h} \cdot \boldsymbol{n} \rrbracket^{2},
$$

for a certain Clément-type quasi-interpolation operator $\tilde{i}$. This motivates the use of the pressure stabilization operator

$$
j\left(p_{h}, q_{h}\right)=\sum_{K \in \mathcal{T}_{h}} \int_{\partial K \backslash \partial \Omega} \frac{h^{3}}{\nu} \llbracket \nabla p_{h} \cdot \boldsymbol{n} \rrbracket \llbracket \nabla q_{h} \cdot \boldsymbol{n} \rrbracket .
$$

Clearly (7) and (8) are verified in this case. Moreover, (11) may be shown to hold if $\mathcal{I}_{h}^{\bar{k}}$ is chosen to be the $L^{2}$-projection onto $\left[V_{h}^{k}\right]^{d}$ and boundary conditions are imposed weakly [13. To show the inequality we combine (18) with (20). Finally, by taking $\Pi_{h}^{k}$ as the $L^{2}$-projection onto $Q_{h}^{k}$, since $\llbracket \mathcal{C}_{h}^{k} \nabla p \rrbracket=\mathbf{0}$ (with $\mathcal{C}_{h}^{k}$ the Clément interpolant onto $\left[X_{h}^{k}\right]^{d}$ ), using an element-wise trace inequality, adding and subracting $\nabla p$, and using the approximation properties of $\mathcal{C}_{h}^{k}$ and $\Pi_{h}^{k}$, one readily verifies (see [13, Lemma 4.7]) the weak consistency

$$
\begin{aligned}
j\left(\Pi_{h}^{k} p, \Pi_{h}^{k} p\right)^{\frac{1}{2}} & \leq C \frac{h}{\nu^{\frac{1}{2}}}\left\|\nabla \Pi_{h}^{k} p-\mathcal{C}_{h}^{k} \nabla p\right\|_{0, \Omega} \\
& \leq \frac{C}{\nu^{\frac{1}{2}}} h^{s_{p}}\|p\|_{s_{p}, \Omega}, \forall p \in H^{s}(\Omega),
\end{aligned}
$$

with $s_{p}=\min \{k+1, s\}$, so that $\tilde{l}=l=k$.

We refer to 13 for the details on the technical issue related to the weak imposition of the boundary conditions using Nitsche's method.

\subsubsection{The Ritz projection operator}

For the purpose of the stability and convergence analysis below we introduce the Ritz projection operator

$$
S_{h}^{k, l}:\left[H^{1}(\Omega)\right]^{d} \times L^{2}(\Omega) \longrightarrow V_{h}^{k} \times Q_{h}^{l} .
$$

For each $(\boldsymbol{u}, p) \in\left[H^{1}(\Omega)\right]^{d} \times L^{2}(\Omega)$, the projection $S_{h}^{k, l}(\boldsymbol{u}, p) \stackrel{\text { def }}{=}\left(P_{h}^{k}(\boldsymbol{u}, p), R_{h}^{l}(\boldsymbol{u}, p)\right) \in$ $\left[V_{h}^{k}\right]^{d} \times Q_{h}^{l}$ is defined as the unique solution of

$$
\left\{\begin{aligned}
a\left(P_{h}^{k}(\boldsymbol{u}, p), \boldsymbol{v}_{h}\right)+b\left(R_{h}^{l}(\boldsymbol{u}, p), \boldsymbol{v}_{h}\right) & =a\left(\boldsymbol{u}, \boldsymbol{v}_{h}\right)+b\left(p, \boldsymbol{v}_{h}\right), \\
-b\left(q_{h}, P_{h}^{k}(\boldsymbol{u}, p)\right)+j\left(R_{h}^{l}(\boldsymbol{u}, p), q_{h}\right) & =0,
\end{aligned}\right.
$$

for all $\left(\boldsymbol{v}_{h}, q_{h}\right) \in\left[V_{h}^{k}\right]^{d} \times Q_{h}^{l}$. 
Problem (21) is well-posed thanks to the inf-sup condition (13), in particular, we have the following a priori stability estimate:

$$
\left\|\left(P_{h}^{k}(\boldsymbol{u}, p), R_{h}^{l}(\boldsymbol{u}, p)\right)\right\|_{h}^{2} \leq C\left(\|\boldsymbol{u}\|_{V}^{2}+\|p\|_{Q}^{2}\right),
$$

with $C>0$ a constant independent of $h$ and $\nu$.

Finally, we have the following approximation result.

Lemma 3.4 Let $\left.(\boldsymbol{u}, p) \in C^{1}\left([0, T],\left[H^{r}(\Omega) \cap H_{0}^{1}(\Omega)\right)\right]^{d} \cap H_{0}(\operatorname{div} ; \Omega) \times H^{s}(\Omega)\right)$ with $r \geq 2$ and $s \geq 1$. The following error estimate for the projection $S_{h}^{k, l}$ holds with $\alpha=0,1$ :

$$
\begin{aligned}
\left\|\left(\partial_{t}^{\alpha}\left(\boldsymbol{u}-P_{h}^{k}(\boldsymbol{u}, p)\right), \partial_{t}^{\alpha} R_{h}^{l}(\boldsymbol{u}, p)\right)\right\|_{h} & \leq C\left(\nu^{\frac{1}{2}} h^{r_{\boldsymbol{u}}-1}\left\|\partial_{t}^{\alpha} \boldsymbol{u}\right\|_{r_{\boldsymbol{u}}, \Omega}+\nu^{-\frac{1}{2}} h^{s_{p}}\left\|\partial_{t}^{\alpha} p\right\|_{s_{p}, \Omega}\right), \\
\left\|p-R_{h}^{l}(\boldsymbol{u}, p)\right\|_{Q} & \leq C\left(\nu^{\frac{1}{2}} h^{r_{u}-1}\|\boldsymbol{u}\|_{r_{\boldsymbol{u}}, \Omega}+\nu^{-\frac{1}{2}} h^{s_{p}}\|p\|_{s_{p}, \Omega}\right),
\end{aligned}
$$

for all $t \in[0, T]$ and with $r_{\boldsymbol{u}} \stackrel{\text { def }}{=} \min \{r, k+1\}, s_{p} \stackrel{\text { def }}{=} \min \{s, \tilde{l}, l+1\}$ and $C>0$ independent of $\nu$ and $h$. Moreover, provided the domain $\Omega$ is sufficiently smooth and if $\tilde{l} \geq 1$, there also holds

$$
\left\|\partial_{t}^{\alpha}\left(\boldsymbol{u}-P_{h}^{k}(\boldsymbol{u}, p)\right)\right\|_{H} \leq C h\left\|\left(\partial_{t}^{\alpha}\left(\boldsymbol{u}-P_{h}^{k}(\boldsymbol{u}, p)\right), \partial_{t}^{\alpha} R_{h}^{l} p\right)\right\|_{h} .
$$

Proof. For simplicity we here use the notation $\boldsymbol{u}_{h} \stackrel{\text { def }}{=} P_{h}^{k}(\boldsymbol{u}, p)$ and $p_{h} \stackrel{\text { def }}{=}$ $R_{h}^{l}(\boldsymbol{u}, p)$. From $(15)$, the $V$-coercivity of $a(\cdot, \cdot)(5)$, and the orthogonality provided by (21) we have

$$
\begin{aligned}
\left\|\left(\boldsymbol{u}_{h}-\mathcal{I}_{h}^{k} \boldsymbol{u}, p_{h}-\Pi_{h}^{l} p\right)\right\|_{h}^{2}= & a\left(\boldsymbol{u}-\mathcal{I}_{h}^{k} \boldsymbol{u}, \boldsymbol{u}_{h}-\mathcal{I}_{h}^{k} \boldsymbol{u}\right)+b\left(p-\Pi_{h}^{l} p, \boldsymbol{u}_{h}-\mathcal{I}_{h}^{k} \boldsymbol{u}\right) \\
& +b\left(p_{h}-\Pi_{h}^{l} p, \boldsymbol{u}-\mathcal{I}_{h}^{k} \boldsymbol{u}\right)+j\left(\Pi_{h}^{l} p, p_{h}-\Pi_{h}^{l} p\right) .
\end{aligned}
$$

Finally, using (5) and (11), we have that

$$
\begin{aligned}
& \left\|\left(\boldsymbol{u}_{h}-\mathcal{I}_{h}^{k} \boldsymbol{u}, p_{h}-\Pi_{h}^{l} p\right)\right\|_{h}^{2} \leq\left(\left\|\boldsymbol{u}-\mathcal{I}_{h}^{k} \boldsymbol{u}\right\|_{V}+\left\|p-\Pi_{h}^{l} p\right\|_{Q}\right)\left\|\boldsymbol{u}_{h}-\mathcal{I}_{h}^{k} \boldsymbol{u}\right\|_{V} \\
+ & C\left(\nu^{\frac{1}{2}}\left\|h^{-1}\left(\boldsymbol{u}-\mathcal{I}_{h}^{k} \boldsymbol{u}\right)\right\|_{H}+\left\|\boldsymbol{u}-\mathcal{I}_{h}^{k} \boldsymbol{u}\right\|_{V}+j\left(\Pi_{h}^{l} p, \Pi_{h}^{l} p\right)^{\frac{1}{2}}\right) j\left(p_{h}-\Pi_{h}^{l} p, p_{h}-\Pi_{l}^{k} p\right)^{\frac{1}{2}}
\end{aligned}
$$

We obtain the estimation for the velocity $(\alpha=0)$ using the approximation properties of $\mathcal{I}_{h}^{k}$ and $\Pi_{h}^{l}(\sqrt{10})$ and $(90)$, and the weak consistency (8) of the stabilizing term $j(\cdot, \cdot)$. The convergence for the time derivative $(\alpha=1)$ is obtained in a similar fashion after time derivation of (21).

For the pressure estimate we use the generalized inf-sup condition 13 and the orthogonality provided by 21 . We then have

$$
\begin{aligned}
\beta\left\|\Pi_{h}^{l} p-p_{h}\right\|_{Q} & \leq \sup _{\boldsymbol{v}_{h} \in\left[V_{h}^{k}\right]^{d}} \frac{b\left(\Pi_{h}^{l} p-p_{h}, \boldsymbol{v}_{h}\right)}{\left\|\boldsymbol{v}_{h}\right\|_{V}}+C j\left(\Pi_{h}^{l} p-p_{h}, \Pi_{h}^{l} p-p_{h}\right)^{\frac{1}{2}} \\
& \leq \sup _{\boldsymbol{v}_{h} \in\left[V_{h}^{k}\right]^{d}} \frac{b\left(\Pi_{h}^{k} p-p, \boldsymbol{v}_{h}\right)-a\left(\boldsymbol{u}-\boldsymbol{u}_{h}, \boldsymbol{v}_{h}\right)}{\left\|\boldsymbol{v}_{h}\right\|_{V}}+C j\left(\Pi_{h}^{l} p-p_{h}, \Pi_{h}^{l} p-p_{h}\right)^{\frac{1}{2}} .
\end{aligned}
$$

We conclude using the continuity of $a(\cdot, \cdot)$ and $b(\cdot, \cdot)$, approximability, the weak consistency of $j(\cdot, \cdot)$ and the previous error estimate. For a proof of the optimality in the $H$-norm we refer e.g. to [13, Theorem 4.14]. 


\subsection{Fully discrete formulation: time discretization}

In this paragraph we discretize $(12)$ with respect to the time variable. To this aim, we will use some known $\mathcal{A}$-stable time discretization schemes for ODE's.

Let $N \in \mathbb{N}^{*}$ be given, we consider a uniform partition $\left\{\left[t_{n}, t_{n+1}\right]\right\}_{0 \leq n \leq N-1}$, with $t_{n} \stackrel{\text { def }}{=} n \delta t$, of the time interval of interest $[0, T]$ with time-step size $\delta t \stackrel{\text { def }}{=}$ $T / N$. The discrete pair $\left(\boldsymbol{u}_{h}^{n}, p_{h}^{n}\right)$ stands for an approximation of $\left(\boldsymbol{u}\left(t_{n}\right), p\left(t_{n}\right)\right)$ in $\left[V_{h}^{k}\right]^{d} \times Q_{h}^{l}$.

\section{Backward Euler scheme (BDF1)}

By introducing the first order backward difference quotient

$$
\bar{D} u_{h}^{n+1} \stackrel{\text { def }}{=} \frac{u_{h}^{n+1}-u_{h}^{n}}{\delta t},
$$

our first fully discrete scheme reads as follows: For $0 \leq n \leq N-1$, find $\left(\boldsymbol{u}_{h}^{n+1}, p_{h}^{n+1}\right) \in\left[V_{h}^{k}\right]^{d} \times Q_{h}^{l}$ such that

$$
\begin{aligned}
\left(\bar{D} \boldsymbol{u}_{h}^{n+1}, \boldsymbol{v}_{h}\right)+a\left(\boldsymbol{u}_{h}^{n+1}, \boldsymbol{v}_{h}\right)+b\left(p_{h}^{n+1}, \boldsymbol{v}_{h}\right) & -b\left(q_{h}, \boldsymbol{u}_{h}^{n+1}\right) \\
& +j\left(p_{h}^{n+1}, q_{h}\right)=\left(\boldsymbol{f}\left(t_{n+1}\right), \boldsymbol{v}_{h}\right),
\end{aligned}
$$

for all $\left(\boldsymbol{v}_{h}, q_{h}\right) \in V_{h}^{k} \times Q_{h}^{l}$ and with $\boldsymbol{u}_{h}^{0}$ a suitable approximation of $\boldsymbol{u}_{0}$ in $\left[V_{h}^{k}\right]^{d}$.

\section{Crank-Nicholson scheme}

Let us consider now the scheme given by: For $0 \leq n \leq N-1$, find $\left(\boldsymbol{u}_{h}^{n+1}, p_{h}^{n+\frac{1}{2}}\right) \in$ $\left[V_{h}^{k}\right]^{d} \times Q_{h}^{l}$ such that

$$
\begin{aligned}
\left(\bar{D} \boldsymbol{u}_{h}^{n+1}, \boldsymbol{v}_{h}\right)+a\left(\boldsymbol{u}_{h}^{n+\frac{1}{2}}, \boldsymbol{v}_{h}\right)+b\left(p_{h}^{n+\frac{1}{2}}, \boldsymbol{v}_{h}\right) & -b\left(q_{h}, \boldsymbol{u}_{h}^{n+\frac{1}{2}}\right) \\
& +j\left(p_{h}^{n+\frac{1}{2}}, q_{h}\right)=\left(\boldsymbol{f}^{n+\frac{1}{2}}, \boldsymbol{v}_{h}\right)
\end{aligned}
$$

for all $\left(\boldsymbol{v}_{h}, q_{h}\right) \in\left[V_{h}^{k}\right]^{d} \times Q_{h}^{l}$, where $\boldsymbol{u}_{h}^{n+\frac{1}{2}} \stackrel{\text { def }}{=} \frac{1}{2}\left(\boldsymbol{u}_{h}^{n+1}+\boldsymbol{u}_{h}^{n}\right)$ and $\boldsymbol{u}_{h}^{0}$ is a suitable approximation of $\boldsymbol{u}_{0}$ in $\left[V_{h}^{k}\right]^{d}$.

Remark 3.5 Note that (25) uniquely determines $\boldsymbol{u}_{h}^{n+1}$, since $\boldsymbol{u}_{h}^{0}$ is given. For the pressure however neither $p_{h}^{n+1}$ nor $p_{h}^{n}$ are used in 25]. Therefore, by working with $p_{h}^{n+\frac{1}{2}}$ as pressure variable, we do not need to provide an initial condition for the pressure. On the other hand, we do not have an approximation of $p_{h}^{n+1}$ unless one is constructed by extrapolation.

\section{Second order backward difference (BDF2)}

Finally, by considering the second order backward difference quotient

$$
\tilde{D} \boldsymbol{u}^{n+1} \stackrel{\text { def }}{=} \frac{1}{2 \delta t}\left(3 \boldsymbol{u}_{h}^{n+1}-4 \boldsymbol{u}_{h}^{n}+\boldsymbol{u}_{h}^{n-1}\right),
$$

we obtain the BDF2 scheme: For $1 \leq n \leq N-1$, find $\left(\boldsymbol{u}_{h}^{n+1}, p_{h}^{n+1}\right) \in\left[V_{h}^{k}\right]^{d} \times Q_{h}^{l}$ such that

$$
\begin{aligned}
\left(\tilde{D} \boldsymbol{u}_{h}^{n+1}, \boldsymbol{v}_{h}\right)+a\left(\boldsymbol{u}_{h}^{n+1}, \boldsymbol{v}_{h}\right)+b\left(p_{h}^{n+1}, \boldsymbol{v}_{h}\right)- & b\left(q_{h}, \boldsymbol{u}_{h}^{n+1}\right) \\
& +j\left(p_{h}^{n+1}, q_{h}\right)=\left(\boldsymbol{f}^{n+1}, \boldsymbol{v}_{h}\right),
\end{aligned}
$$


for all $\left(\boldsymbol{v}_{h}, q_{h}\right) \in\left[V_{h}^{k}\right]^{d} \times Q_{h}^{l}$, and $\left(\boldsymbol{u}_{h}^{1}, p_{h}^{1}\right) \in\left[V_{h}^{k}\right]^{d} \times Q_{h}^{l}$ given by the first step of backward Euler scheme (24).

\section{$4 \quad$ Stability}

In this section we analyze the stability properties of the fully discrete schemes introduced in 3.2 For the sake of simplicity, full details will be only given for the backward scheme (24). Nevertheless, in $\$ 4.2$ we will discuss how the results extend to the second order time stepping schemes: Crank-Nicholson and BDF2.

\subsection{First order $\mathcal{A}$-stable scheme}

The next result provides the unconditional stability of the velocity. It also provides a uniform estimate for the pressure, in terms of the discrete velocity time derivative. Theorem 4.2 points out the role of the initial velocity approximation on the stability of the velocity time derivative approximations. Finally, Corollary 4.3 states the (conditional or unconditional) stability of the pressure depending on the choice of the initial velocity approximation.

Theorem 4.1 Let $\boldsymbol{u}_{h}^{0}$ be a given $H$-stable approximation of $\boldsymbol{u}_{0}$ in $\left[V_{h}^{k}\right]^{d}$ and $\left\{\left(\boldsymbol{u}_{h}^{n}, p_{h}^{n}\right)\right\}_{n=1}^{N}$ be the solution of the fully discrete problem (24). The following estimate holds for $1 \leq n \leq N$ :

$$
\begin{aligned}
& \left\|\boldsymbol{u}_{h}^{n}\right\|_{H}^{2}+\sum_{m=0}^{n-1} \delta t\left\|\left(\boldsymbol{u}_{h}^{m+1}, p_{h}^{m+1}\right)\right\|_{h}^{2} \leq C\left\|\boldsymbol{u}_{0}\right\|_{H}^{2}+\frac{C_{\mathrm{P}}^{2}}{\nu} \sum_{m=0}^{n-1} \delta t\left\|\boldsymbol{f}\left(t_{m+1}\right)\right\|_{H}^{2}, \\
& \sum_{m=0}^{n-1} \delta t\left\|p_{h}^{m+1}\right\|_{Q}^{2} \leq \frac{C}{\beta^{2}} \sum_{m=0}^{n-1} \delta t\left(\left\|\left(\boldsymbol{u}_{h}^{m+1}, p_{h}^{m+1}\right)\right\|_{h}^{2}+\nu^{-1}\left\|\bar{D} \boldsymbol{u}_{h}^{m+1}\right\|_{H}^{2}+\nu^{-1}\left\|\boldsymbol{f}\left(t_{m+1}\right)\right\|_{H}^{2}\right) .
\end{aligned}
$$

with $C_{\mathrm{P}}>0$ the Poincaré's constant.

Proof. Taking $\boldsymbol{v}_{h}=\boldsymbol{u}_{h}^{n+1}$ and $q_{h}=p_{h}^{n+1}$ in (24), using the coercivity of the bi-linear form, the Cauchy-Schwarz's inequality and the Poincaré's inequality, we have

$$
\left(\bar{D} \boldsymbol{u}_{h}^{n+1}, \boldsymbol{u}_{h}^{n+1}\right)+\frac{1}{2}\left\|\left(\boldsymbol{u}_{h}^{n+1}, p_{h}^{n+1}\right)\right\|_{h}^{2} \leq \frac{C_{\mathrm{P}}^{2}}{2 \nu}\left\|\boldsymbol{f}\left(t_{n+1}\right)\right\|_{H}^{2} .
$$

Now, recalling that

$$
\left(\bar{D} \boldsymbol{u}_{h}^{n+1}, \boldsymbol{u}_{h}^{n+1}\right)=\frac{1}{2} \bar{D}\left\|\boldsymbol{u}_{h}^{n+1}\right\|_{H}^{2}+\frac{1}{2 \delta t}\left\|\boldsymbol{u}_{h}^{n+1}-\boldsymbol{u}_{h}^{n}\right\|_{H}^{2},
$$

we have

$$
\bar{D}\left\|\boldsymbol{u}_{h}^{n+1}\right\|_{H}^{2}+\left\|\left(\boldsymbol{u}_{h}^{n+1}, p_{h}^{n+1}\right)\right\|_{h}^{2} \leq \frac{C_{\mathrm{P}}^{2}}{\nu}\left\|\boldsymbol{f}\left(t_{n+1}\right)\right\|_{H}^{2},
$$

leading to, after summation over $0 \leq m \leq n-1$,

$$
\left\|\boldsymbol{u}_{h}^{n}\right\|_{H}^{2}+\sum_{m=0}^{n-1} \delta t\left\|\left(\boldsymbol{u}_{h}^{m+1}, p_{h}^{m+1}\right)\right\|_{h}^{2} \leq\left\|\boldsymbol{u}_{h}^{0}\right\|_{H}^{2}+\frac{C_{\mathrm{P}}^{2}}{\nu} \sum_{m=0}^{n-1} \delta t\left\|\boldsymbol{f}\left(t_{m+1}\right)\right\|_{H}^{2} .
$$


For the pressure estimate, from 13 , 24) (with $q_{h}=0$ ) and the Poincaré's inequality, we have

$$
\beta\left\|p_{h}^{n+1}\right\|_{Q} \leq C\left(\left\|\left(\boldsymbol{u}_{h}^{n+1}, p_{h}^{n+1}\right)\right\|_{h}+\nu^{-\frac{1}{2}}\left\|\bar{D} \boldsymbol{u}_{h}^{n+1}\right\|_{H}+\nu^{-\frac{1}{2}}\left\|\boldsymbol{f}\left(t_{n+1}\right)\right\|_{H}\right),
$$

which completes the proof. $\square$

The next theorem states some a priori estimates of the approximations of the velocity time derivative.

Theorem 4.2 Let $\left\{\left(\boldsymbol{u}_{h}^{n}, p_{h}^{n}\right)\right\}_{n=1}^{N}$ be the solution of the fully discrete problem (24).

- If $\boldsymbol{u}_{0} \in\left[H^{1}(\Omega)\right]^{d}$ and $\boldsymbol{u}_{h}^{0}=P_{h}^{k}\left(\boldsymbol{u}_{0}, 0\right)$, the following estimate holds for $1 \leq n \leq N$ :

$$
\sum_{m=0}^{n-1} \delta t\left\|\bar{D} \boldsymbol{u}_{h}^{m+1}\right\|_{H}^{2}+\left\|\left(\boldsymbol{u}_{h}^{n}, p_{h}^{n}\right)\right\|_{h}^{2} \leq C\left(\left\|\boldsymbol{u}_{0}\right\|_{V}^{2}+\sum_{m=0}^{n-1} \delta t\left\|\boldsymbol{f}\left(t_{m+1}\right)\right\|_{H}^{2}\right) .
$$

- If $\boldsymbol{u}_{0} \in\left[H^{r}(\Omega) \cap H_{0}^{1}(\Omega)\right]^{d} \cap H_{0}(\operatorname{div} ; \Omega), r \geq 2$, and $\boldsymbol{u}_{h}^{0}=\mathcal{I}_{h}^{k} \boldsymbol{u}_{0}$, the following estimate holds for $1 \leq n \leq N$ :

$$
\begin{array}{r}
\sum_{m=0}^{n-1} \delta t\left\|\bar{D} \boldsymbol{u}_{h}^{m+1}\right\|_{H}^{2}+\left\|\left(\boldsymbol{u}_{h}^{n}, p_{h}^{n}\right)\right\|_{h}^{2} \leq C\left(\left\|\boldsymbol{u}_{0}\right\|_{V}^{2}+\nu h^{2\left(r_{\boldsymbol{u}}-1\right)}\left\|p_{h}^{1}\right\|_{Q}^{2}\right. \\
\left.+\left\|\boldsymbol{u}_{0}\right\|_{r_{\boldsymbol{u}}, \Omega}^{2}+\sum_{m=0}^{n-1} \delta t\left\|\boldsymbol{f}\left(t_{m+1}\right)\right\|_{H}^{2}\right)
\end{array}
$$

with $r_{\boldsymbol{u}} \stackrel{\text { def }}{=} \min \{k+1, r\}$.

Proof. For $0 \leq n \leq N-1$, by taking $\boldsymbol{v}_{h}=\bar{D} \boldsymbol{u}_{h}^{n+1}$ and $q_{h}=0$ in (24) and using the Cauchy-Schwarz inequality, we have

$$
\frac{1}{2}\left\|\bar{D} \boldsymbol{u}_{h}^{n+1}\right\|_{H}^{2}+a\left(\boldsymbol{u}_{h}^{n+1}, \bar{D} \boldsymbol{u}_{h}^{n+1}\right)+b\left(p_{h}^{n+1}, \bar{D} \boldsymbol{u}_{h}^{n+1}\right)=\frac{1}{2}\left\|\boldsymbol{f}\left(t_{n+1}\right)\right\|_{H}^{2},
$$

On the other hand, for $1 \leq n \leq N-1$, testing (24) at the time levels $n$ and $n+1$ with $\boldsymbol{v}_{h}=\mathbf{0}$ and $q_{h}=p_{h}^{n+1}$ we have

$$
\begin{aligned}
b\left(p_{h}^{n+1}, \boldsymbol{u}_{h}^{n+1}\right) & =j\left(p_{h}^{n+1}, p_{h}^{n+1}\right), \\
b\left(p_{h}^{n+1}, \boldsymbol{u}_{h}^{n}\right) & =j\left(p_{h}^{n}, p_{h}^{n+1}\right) .
\end{aligned}
$$

Therefore, by subtracting these equalities and using the bi-linearity of $j(\cdot, \cdot)$, we obtain

$$
b\left(p_{h}^{n+1}, \bar{D} \boldsymbol{u}_{h}^{n+1}\right)=j\left(\bar{D} p_{h}^{n+1}, p_{h}^{n+1}\right),
$$

for $1 \leq n \leq N-1$. It then follows, from (32), that

$$
\frac{1}{2}\left\|\bar{D} \boldsymbol{u}_{h}^{n+1}\right\|_{H}^{2}+a\left(\boldsymbol{u}_{h}^{n+1}, \bar{D} \boldsymbol{u}_{h}^{n+1}\right)+j\left(p_{h}^{n+1}, \bar{D} p_{h}^{n+1}\right) \leq \frac{1}{2}\left\|\boldsymbol{f}\left(t_{n+1}\right)\right\|_{H}^{2} .
$$


On the other hand, using the symmetry and bi-linearity of $a(\cdot, \cdot)$ and $j(\cdot, \cdot)$, we have

$$
\begin{aligned}
a\left(\boldsymbol{u}_{h}^{n+1}, \bar{D} \boldsymbol{u}_{h}^{n+1}\right) & =\frac{1}{2} \bar{D} a\left(\boldsymbol{u}_{h}^{n+1}, \boldsymbol{u}_{h}^{n+1}\right)+\frac{\delta t}{2} a\left(\bar{D} \boldsymbol{u}_{h}^{n+1}, \bar{D} \boldsymbol{u}_{h}^{n+1}\right), \\
j\left(p_{h}^{n+1}, \bar{D} p_{h}^{n+1}\right) & =\frac{1}{2} \bar{D} j\left(p_{h}^{n+1}, p_{h}^{n+1}\right)+\frac{\delta t}{2} j\left(\bar{D} p_{h}^{n+1}, \bar{D} p_{h}^{n+1}\right) .
\end{aligned}
$$

Hence,

$$
\left\|\bar{D} \boldsymbol{u}_{h}^{n+1}\right\|_{H}^{2}+\bar{D}\left(a\left(\boldsymbol{u}_{h}^{n+1}, \boldsymbol{u}_{h}^{n+1}\right)+j\left(p_{h}^{n+1}, p_{h}^{n+1}\right)\right) \leq\left\|\boldsymbol{f}\left(t_{n+1}\right)\right\|_{H}^{2},
$$

for $1 \leq n \leq N-1$. After multiplication by $\delta t$ and summation over $1 \leq m \leq n-1$, it follows that

$$
\sum_{m=1}^{n-1} \delta t\left\|\bar{D} \boldsymbol{u}_{h}^{m+1}\right\|_{H}^{2}+\left\|\left(\boldsymbol{u}_{h}^{n}, p_{h}^{n}\right)\right\|_{h}^{2} \leq\left\|\left(\boldsymbol{u}_{h}^{1}, p_{h}^{1}\right)\right\|_{h}^{2}+\sum_{m=1}^{n-1} \delta t\left\|\boldsymbol{f}\left(t_{m+1}\right)\right\|_{H}^{2} .
$$

In order to highlight the impact of the initial velocity approximation on the stability of the time derivative, we consider now the first time level $(n=0)$ of (24). By testing with $\boldsymbol{v}_{h}=\bar{D} \boldsymbol{u}_{h}^{1}, q_{h}=0$, after multiplication by $2 \delta t$ and using the symmetry and bi-linearity of $a(\cdot, \cdot)$, we get

$$
\delta t\left\|\bar{D} \boldsymbol{u}_{h}^{1}\right\|_{H}^{2}+a\left(\boldsymbol{u}_{h}^{1}, \boldsymbol{u}_{h}^{1}\right)-a\left(\boldsymbol{u}_{h}^{0}, \boldsymbol{u}_{h}^{0}\right)+2 \delta t b\left(p_{h}^{1}, \bar{D} \boldsymbol{u}_{h}^{1}\right) \leq \delta t\left\|\boldsymbol{f}\left(t_{1}\right)\right\|_{H}^{2} .
$$

If the initial velocity approximation is given in terms of the Ritz-projection, $\boldsymbol{u}_{h}^{0}=P_{h}^{k}\left(\boldsymbol{u}_{0}, 0\right)$ with $\boldsymbol{u}_{0} \in\left[H^{1}(\Omega)\right]^{d}$, by setting $p_{h}^{0} \stackrel{\text { def }}{=} R_{h}^{l}\left(\boldsymbol{u}_{0}, 0\right)$ it follows that (33) also holds for $n=0$. Therefore,

$$
b\left(p_{h}^{1}, \bar{D} \boldsymbol{u}_{h}^{1}\right)=j\left(\bar{D} p_{h}^{1}, p_{h}^{1}\right),
$$

Thus, from the symmetry and bi-linearity of $j(\cdot, \cdot)$ and (37), we have

$$
\delta t\left\|\bar{D} \boldsymbol{u}_{h}^{1}\right\|_{H}^{2}+\left\|\left(\boldsymbol{u}_{h}^{1}, p_{h}^{1}\right)\right\|_{h}^{2} \leq\left\|\left(\boldsymbol{u}_{h}^{0}, p_{h}^{0}\right)\right\|_{h}^{2}+\delta t\left\|\boldsymbol{f}\left(t_{1}\right)\right\|_{H}^{2} .
$$

Estimate (30) is obtained by adding this last inequality to (36) and using the stability of the Ritz-projection $222,\|\|\left(\boldsymbol{u}_{h}^{0}, p_{h}^{0}\right)\left\|_{h}^{2} \leq C\right\| \boldsymbol{u}_{0} \|_{V}^{2}$.

If the initial velocity approximation is given in terms of a general interpolant, $\boldsymbol{u}_{h}^{0}=\mathcal{I}_{h}^{k} \boldsymbol{u}_{0}$ with $\boldsymbol{u}_{0} \in\left[H^{r}(\Omega) \cap H_{0}^{1}(\Omega)\right]^{d} \cap H_{0}$ (div; $\Omega$ ), equality (38) does not hold in general. Instead, we can use an approximation argument to obtain

$$
\begin{aligned}
b\left(p_{h}^{1}, \bar{D} \boldsymbol{u}_{h}^{1}\right) & =\frac{1}{\delta t}\left(j\left(p_{h}^{1}, p_{h}^{1}\right)-\left(p_{h}^{1}, \boldsymbol{\nabla} \cdot\left(\mathcal{I}_{h}^{k} \boldsymbol{u}_{0}-\boldsymbol{u}_{0}\right)\right)\right) \\
& \geq \frac{1}{\delta t} j\left(p_{h}^{1}, p_{h}^{1}\right)-\frac{C_{\mathcal{I}}}{\delta t}\left(\nu h^{2\left(r_{\boldsymbol{u}}-1\right)}\left\|p_{h}^{1}\right\|_{Q}^{2}+\left\|\boldsymbol{u}_{0}\right\|_{r_{\boldsymbol{u}}, \Omega}^{2}\right),
\end{aligned}
$$

with $r_{\boldsymbol{u}} \stackrel{\text { def }}{=} \min \{k+1, r\}$. As a result, from (37), it follows that

$$
\begin{aligned}
\delta t\left\|\bar{D} \boldsymbol{u}_{h}^{1}\right\|_{H}^{2}+\left\|\left(\boldsymbol{u}_{h}^{1}, p_{h}^{1}\right)\right\|_{h}^{2} \leq a\left(\boldsymbol{u}_{h}^{0}, \boldsymbol{u}_{h}^{0}\right)+C_{\mathcal{I}}\left(\nu h^{2\left(r_{u}-1\right)}\left\|p_{h}^{1}\right\|_{Q}^{2}\right. & \left.+\left\|\boldsymbol{u}_{0}\right\|_{r_{u}, \Omega}^{2}\right) \\
& +\delta t\left\|\boldsymbol{f}\left(t_{1}\right)\right\|_{H}^{2} .
\end{aligned}
$$

We conclude the proof by adding this equality to 36 and using the stability of the Ritz-projection.

The next Corollary closes the problem of the stability of the pressures by combining the results of Theorems 4.1 and 4.2 . 
Corollary 4.3 Let $\left\{\left(\boldsymbol{u}_{h}^{n}, p_{h}^{n}\right)\right\}_{n=1}^{N}$ be the solution of the fully discrete problem (24). Then:

- If $\boldsymbol{u}_{0} \in\left[H^{1}(\Omega)\right]^{d}$ and $\boldsymbol{u}_{h}^{0}=P_{h}^{k}\left(\boldsymbol{u}_{0}, 0\right)$, the following estimate holds for $1 \leq n \leq N$ :

$$
\begin{aligned}
\sum_{m=0}^{n-1} \delta t\left\|p_{h}^{m+1}\right\|_{Q}^{2} & \leq \frac{C}{\beta^{2} \nu}\left\|\boldsymbol{u}_{0}\right\|_{V}^{2} \\
+ & \frac{C}{\beta^{2}} \sum_{m=0}^{n-1} \delta t\left(\left\|\left(\boldsymbol{u}_{h}^{m+1}, p_{h}^{m+1}\right)\right\|_{h}^{2}+\nu^{-1}\left\|\boldsymbol{f}\left(t_{m+1}\right)\right\|_{H}^{2}\right) .
\end{aligned}
$$

- If $\boldsymbol{u}_{0} \in\left[H^{r}(\Omega) \cap H_{0}^{1}(\Omega)\right]^{d} \cap H_{0}(\operatorname{div} ; \Omega), r \geq 2, \boldsymbol{u}_{h}^{0}=\mathcal{I}_{h}^{k} \boldsymbol{u}_{0}$ and

$$
\frac{2 C_{\mathcal{I}}}{\beta^{2}} h^{2\left(r_{u}-1\right)} \leq \delta t,
$$

the following estimate holds for $1 \leq n \leq N$ :

$$
\begin{aligned}
\sum_{m=0}^{n-1} \delta t\left\|p_{h}^{m+1}\right\|_{Q}^{2} & \leq \frac{C}{\beta^{2} \nu}\left(\left\|\boldsymbol{u}_{0}\right\|_{V}^{2}+\left\|\boldsymbol{u}_{0}\right\|_{r_{u}, \Omega}^{2}\right) \\
+ & \frac{C}{\beta^{2}} \sum_{m=0}^{n-1} \delta t\left(\left\|\left(\boldsymbol{u}_{h}^{m+1}, p_{h}^{m+1}\right)\right\|_{h}^{2}+\nu^{-1}\left\|\boldsymbol{f}\left(t_{m+1}\right)\right\|_{H}^{2}\right),
\end{aligned}
$$

with $r_{\boldsymbol{u}} \stackrel{\text { def }}{=} \min \{k+1, r\}$.

Proof. Estimate (43) is a direct consequence of Theorem 4.1 and estimate (30). On the other hand, from Theorem 4.1 and estimate [31), we have

$$
\begin{aligned}
& \left(\beta^{2} \delta t-C_{\mathcal{I}} h^{2\left(r_{\boldsymbol{u}}-1\right)}\right)\left\|p_{h}^{1}\right\|_{Q}^{2}+\beta^{2} \sum_{m=1}^{n-1} \delta t\left\|p_{h}^{m+1}\right\|_{Q}^{2} \\
\leq & \frac{C}{\nu}\left(\left\|\boldsymbol{u}_{0}\right\|_{V}^{2}+\left\|\boldsymbol{u}_{0}\right\|_{r_{u}, \Omega}^{2}\right)+C \sum_{m=0}^{n-1} \delta t\left(\left\|\left(\boldsymbol{u}_{h}^{m+1}, p_{h}^{m+1}\right)\right\|_{h}^{2}+\nu^{-1}\left\|\boldsymbol{f}\left(t_{m+1}\right)\right\|_{H}^{2}\right),
\end{aligned}
$$

which combined with the stability condition 42 leads to 43 .

A few observations are now in order. Corollary 4.3 states the unconditional stability of the pressure provided the initial velocity approximation $\boldsymbol{u}_{h}^{0}$ is given in terms of the Ritz-projection operator (21). In the general case, i.e. whenever $\boldsymbol{u}_{h}^{0}$ does not satisfy a discrete divergence free condition (as $\boldsymbol{u}_{h}^{1}$ does), only conditional stability can be guaranteed. As a matter of fact, from the stability condition (42), pressure instabilities are expected for very small time steps. This issue will be illustrated by numerical experiments in section $\$ 6$.

Finally, let us mention that residual based stabilization methods, such as PSPG and GaLS, combined with finite differences time discretization schemes, are known to give rise to pressure instabilities in the small time step limit, see [3, 19. Indeed, it has been shown in [3] that the finite difference/pressure coupling of the stabilization perturbs the coercivity of the discrete pressure operator unless a condition of the type

$$
C h^{2} \leq \delta t,
$$


is satisfied. It is worth emphasizing that, although the stability conditions (44) and (42) are somehow similar, their nature is different. Actually, the instabilities anticipated by Corollary 4.3 are related to the discrete divergence free character of the initial velocity approximation, but not to the structure of the pressure stabilization $j(\cdot, \cdot)$.

\subsection{Second order $\mathcal{A}$-stable schemes}

In this paragraph we discuss how the results of Theorems 4.1 and 4.2 and Corollary 4.3 extend to the second order time stepping schemes Crank-Nicholson and BDF2.

\section{Crank-Nicholson}

The following theorem summarizes the resulting stability estimates.

Theorem 4.4 Let $\boldsymbol{u}_{h}^{0}$ be a given $H$-stable approximation of $\boldsymbol{u}_{0}$ in $\left[V_{h}^{k}\right]^{d}$ and $\left\{\left(\boldsymbol{u}_{h}^{n}, p_{h}^{n}\right)\right\}_{n=1}^{N}$ be the solution of the discrete scheme 25]. Then the following estimate holds for $1 \leq n \leq N$ :

$$
\left\|\boldsymbol{u}_{h}^{n}\right\|_{H}^{2}+\sum_{m=0}^{n-1} \delta t\left\|\left(\boldsymbol{u}_{h}^{m+\frac{1}{2}}, p_{h}^{m+\frac{1}{2}}\right)\right\|_{h}^{2} \leq C\left\|\boldsymbol{u}_{0}\right\|_{H}^{2}+\frac{C_{\mathrm{P}}^{2}}{\nu} \sum_{m=0}^{n-1} \delta t\left\|\boldsymbol{f}\left(t_{m+\frac{1}{2}}\right)\right\|_{H}^{2} .
$$

Moreover, if $\boldsymbol{u}_{0} \in\left[H^{1}(\Omega)\right]^{d}$ and $\boldsymbol{u}_{h}^{0}=P_{h}^{k}\left(\boldsymbol{u}_{0}, 0\right)$, the following estimate holds for $1 \leq n \leq N$ :

$\sum_{m=0}^{n-1} \delta t\left\|p_{h}^{m+\frac{1}{2}}\right\|_{Q}^{2} \leq \frac{C}{\beta^{2} \nu}\left\|\boldsymbol{u}_{0}\right\|_{V}^{2}+\frac{C}{\beta^{2}} \sum_{m=0}^{n-1} \delta t\left(\left\|\left(\boldsymbol{u}_{h}^{m+\frac{1}{2}}, p_{h}^{m+\frac{1}{2}}\right)\right\|_{h}^{2}+\nu^{-1}\left\|\boldsymbol{f}\left(t_{m+\frac{1}{2}}\right)\right\|_{H}^{2}\right)$.

On the other hand, if $\boldsymbol{u}_{0} \in\left[H^{r}(\Omega) \cap H_{0}^{1}(\Omega)\right]^{d} \cap H_{0}(\operatorname{div} ; \Omega), r \geq 2, \boldsymbol{u}_{h}^{0}=\mathcal{I}_{h}^{k} \boldsymbol{u}_{0}$ and the stability condition (42) is satisfied, the following estimate holds for $1 \leq n \leq N$ :

$$
\begin{aligned}
\sum_{m=0}^{n-1} \delta t\left\|p_{h}^{m+\frac{1}{2}}\right\|_{Q}^{2} \leq & \frac{C}{\beta^{2} \nu}\left(\left\|\boldsymbol{u}_{0}\right\|_{V}^{2}+\left\|\boldsymbol{u}_{0}\right\|_{r_{\boldsymbol{u}}, \Omega}^{2}\right) \\
& +\frac{C}{\beta^{2}} \sum_{m=0}^{n-1} \delta t\left(\left\|\left(\boldsymbol{u}_{h}^{m+\frac{1}{2}}, p_{h}^{m+\frac{1}{2}}\right)\right\|_{h}^{2}+\nu^{-1}\left\|\boldsymbol{f}\left(t_{m+\frac{1}{2}}\right)\right\|_{H}^{2}\right) .
\end{aligned}
$$

Proof. The first estimate, corresponding to Theorem 4.1, holds by taking $\boldsymbol{v}_{h}=\boldsymbol{u}_{h}^{n+\frac{1}{2}}$ and $q_{h}=p_{h}^{n+\frac{1}{2}}$ in 25 .

The pressure estimate requires an a priori bound of the discrete velocity time derivative. As in Theorem 4.2, such an estimate can be obtained by taking $\boldsymbol{v}_{h}=\bar{D} \boldsymbol{u}_{h}^{n+1}$ and $q_{h}=0$ in 25 , for $0 \leq n \leq N-1$. The main difference, with respect to the proof of Theorem 4.2 , arises in the treatment of the coupling term $b\left(p_{h}^{n+\frac{1}{2}}, \bar{D} \boldsymbol{u}_{h}^{n+1}\right)$. Indeed, in the Crank-Nicholson scheme incompressibility is enforced on $\boldsymbol{u}_{h}^{n+\frac{1}{2}}$ instead of $\boldsymbol{u}_{h}^{n+1}$. We first note that, since

$$
\boldsymbol{u}_{h}^{n+1}-\boldsymbol{u}_{h}^{n}=2\left(\boldsymbol{u}_{h}^{n+\frac{1}{2}}-\boldsymbol{u}_{h}^{n}\right), \quad \boldsymbol{u}_{h}^{n}=2 \boldsymbol{u}_{h}^{n-1+\frac{1}{2}}-\boldsymbol{u}_{h}^{n-1},
$$


we have

$$
\boldsymbol{u}_{h}^{n+1}-\boldsymbol{u}_{h}^{n}=2 \boldsymbol{u}_{h}^{n+\frac{1}{2}}+4 \sum_{l=1}^{n}(-1)^{l} \boldsymbol{u}_{h}^{n-l+\frac{1}{2}}-(-1)^{n} 2 \boldsymbol{u}_{h}^{0},
$$

for $0 \leq n \leq N-1$. Therefore, from (25) and using the bi-linearity of $j(\cdot, \cdot)$, we get

$$
\begin{aligned}
b\left(p_{h}^{n+\frac{1}{2}}, \boldsymbol{u}_{h}^{n+1}-\boldsymbol{u}_{h}^{n}\right)= & j\left(2 p_{h}^{n+\frac{1}{2}}+4 \sum_{l=1}^{n}(-1)^{l} p_{h}^{n-l+\frac{1}{2}}, p_{h}^{n+\frac{1}{2}}\right) \\
& -2(-1)^{n} b\left(p_{h}^{n+\frac{1}{2}}, \boldsymbol{u}_{h}^{0}\right) .
\end{aligned}
$$

On the other hand, for $0 \leq n \leq N-1$, we introduce the following change of variables (or extrapolation)

$$
\frac{1}{2}\left(p_{h}^{n+1}+p_{h}^{n}\right) \stackrel{\text { def }}{=} p_{h}^{n+\frac{1}{2}},
$$

with $p_{h}^{0} \in Q_{h}^{l}$ to be specified later on. By inserting this expression in 45 we obtain

$$
\begin{aligned}
b\left(p_{h}^{n+\frac{1}{2}}, \boldsymbol{u}_{h}^{n+1}-\boldsymbol{u}_{h}^{n}\right)= & j\left(p_{h}^{n+1}-p_{h}^{n}, p_{h}^{n+\frac{1}{2}}\right) \\
& +2(-1)^{n}\left[j\left(p_{h}^{0}, p_{h}^{n+\frac{1}{2}}\right)-b\left(p_{h}^{n+\frac{1}{2}}, \boldsymbol{u}_{h}^{0}\right)\right] .
\end{aligned}
$$

If $\boldsymbol{u}_{h}^{0}=P_{h}^{k}\left(\boldsymbol{u}_{0}, 0\right)$ and we choose $p_{h}^{0} \stackrel{\text { def }}{=} R_{h}^{l}\left(\boldsymbol{u}_{0}, 0\right)$, from 21$)_{2}$ it follows that the last term in (46) cancels. Thus, we have

$$
\begin{aligned}
b\left(p_{h}^{n+\frac{1}{2}}, \bar{D} \boldsymbol{u}_{h}^{n+1}\right) & =j\left(\bar{D} p_{h}^{n+1}, p_{h}^{n+\frac{1}{2}}\right) \\
& =\frac{1}{2} \bar{D} j\left(p_{h}^{n+1}, p_{h}^{n+1}\right),
\end{aligned}
$$

which corresponds to the Crank-Nicholson's counterpart of (34).

Finally, when the initial velocity approximation is given in terms of a general interpolant, $\boldsymbol{u}_{h}^{0}=\mathcal{I}_{h}^{k} \boldsymbol{u}_{0}$ with $\boldsymbol{u}_{0} \in\left[H^{r}(\Omega) \cap H_{0}^{1}(\Omega)\right]^{d} \cap H_{0}(\operatorname{div} ; \Omega)$, we take $p_{h}^{0} \stackrel{\text { def }}{=} 0$. Therefore, from (46) and using an approximation argument (as in (40)), we get

$$
\begin{aligned}
b\left(p_{h}^{n+\frac{1}{2}}, \bar{D} \boldsymbol{u}_{h}^{n+1}\right)= & \frac{1}{2} \bar{D} j\left(p_{h}^{n+1}, p_{h}^{n+1}\right)-\frac{2}{\delta t}(-1)^{n} b\left(p_{h}^{n+\frac{1}{2}}, \boldsymbol{u}_{h}^{0}\right) \\
\geq & \frac{1}{2} \bar{D} j\left(p_{h}^{n+1}, p_{h}^{n+1}\right) \\
& -\frac{2 C_{\mathcal{I}}}{\delta t}\left(\nu h^{2\left(r_{\boldsymbol{u}}-1\right)}\left\|p_{h}^{n+\frac{1}{2}}\right\|_{Q}^{2}+\left\|\boldsymbol{u}_{0}\right\|_{r_{\boldsymbol{u}}, \Omega}^{2}\right),
\end{aligned}
$$

which leads to the stability condition $(42)$. The rest of the proof follows with minor modifications.

Remark 4.5 By comparing the proofs of Corollary 4.3 and the previous Theorem we can notice that, if the initial velocity approximation is not discretely divergence free, the stability condition (42) has to be satisfied at each time level when using the Crank-Nicholson scheme (due to (47)), whereas for the Backward Euler scheme that condition is only needed at the first time step (thanks to (34) and (40)). 


\section{Second order backward difference}

The following theorem summarizes the resulting stability estimates.

Theorem 4.6 Let $\boldsymbol{u}_{h}^{0}$ be a given $H$-stable approximation of $\boldsymbol{u}_{0}$ in $\left[V_{h}^{k}\right]^{d},\left(u_{h}^{1}, p_{h}^{1}\right)$ be the corresponding first time step of the Backward Euler scheme (24) and $\left\{\left(\boldsymbol{u}_{h}^{n}, p_{h}^{n}\right)\right\}_{n=2}^{N}$ be the solution of the discrete scheme (26). Then, the following estimate holds for $2 \leq n \leq N$ :

$\left\|\boldsymbol{u}_{h}^{n}\right\|_{H}^{2}+2 \sum_{m=1}^{n-1} \delta t\left\|\left(\boldsymbol{u}_{h}^{m+1}, p_{h}^{m+1}\right)\right\|_{h}^{2} \leq C\left(\left\|\boldsymbol{u}_{0}\right\|_{H}^{2}+\left\|\boldsymbol{u}_{h}^{1}\right\|_{H}^{2}\right)+\frac{2 C_{\mathrm{P}}^{2}}{\nu} \sum_{m=1}^{n-1} \delta t\left\|\boldsymbol{f}\left(t_{m+1}\right)\right\|_{H}^{2}$.

Moreover, if $\boldsymbol{u}_{0} \in\left[H^{1}(\Omega)\right]^{d}$ and $\boldsymbol{u}_{h}^{0}=P_{h}^{k}\left(\boldsymbol{u}_{0}, 0\right)$, the following estimate holds for $2 \leq n \leq N$ :

$$
\begin{aligned}
\sum_{m=1}^{n-1} \delta t\left\|p_{h}^{m+1}\right\|_{Q}^{2} \leq \frac{C}{\beta^{2} \nu} & \left(\left\|\boldsymbol{u}_{0}\right\|_{V}^{2}+\left\|\left(\boldsymbol{u}_{h}^{1}, p_{h}^{1}\right)\right\|_{h}^{2}\right) \\
& +\frac{C}{\beta^{2}} \sum_{m=1}^{n-1} \delta t\left(\left\|\left(\boldsymbol{u}_{h}^{m+1}, p_{h}^{m+1}\right)\right\|_{h}^{2}+\nu^{-1}\left\|\boldsymbol{f}\left(t_{m+1}\right)\right\|_{H}^{2}\right) .
\end{aligned}
$$

On the other hand, if $\boldsymbol{u}_{0} \in\left[H^{r}(\Omega) \cap H_{0}^{1}(\Omega)\right]^{d} \cap H_{0}(\operatorname{div} ; \Omega), r \geq 2, \boldsymbol{u}_{h}^{0}=\mathcal{I}_{h}^{k} \boldsymbol{u}_{0}$ and the stability condition (42) is satisfied, the following estimate holds for $2 \leq n \leq N$ :

$$
\begin{aligned}
\sum_{m=1}^{n-1} \delta t\left\|p_{h}^{m+1}\right\|_{Q}^{2} \leq \frac{C}{\beta^{2} \nu} & \left(\left\|\boldsymbol{u}_{0}\right\|_{V}^{2}+\left\|\boldsymbol{u}_{0}\right\|_{r_{\boldsymbol{u}}, \Omega}^{2}+\left\|\left(\boldsymbol{u}_{h}^{1}, p_{h}^{1}\right)\right\|_{h}^{2}\right) \\
& +\frac{C}{\beta^{2}} \sum_{m=1}^{n-1} \delta t\left(\left\|\left(\boldsymbol{u}_{h}^{m+1}, p_{h}^{m+1}\right)\right\|_{h}^{2}+\nu^{-1}\left\|\boldsymbol{f}\left(t_{m+1}\right)\right\|_{H}^{2}\right) .
\end{aligned}
$$

Proof. The first estimate, corresponding to Theorem 4.1, holds by taking $\boldsymbol{v}_{h}=\boldsymbol{u}_{h}^{n+1}$ and $q_{h}=p_{h}^{n+1}$ in 26 and applying the standard identity

$$
(3 a-4 b+c) a=\frac{1}{2}\left[a^{2}-b^{2}+(2 a-b)^{2}-(2 b-c)^{2}+(a-2 b+c)^{2}\right] .
$$

providing the numerical dissipation of the BDF2 scheme.

Since the pressure estimate is based here on the control of the time derivative, $\tilde{D} \boldsymbol{u}_{h}^{n+1}$, we take $\boldsymbol{v}_{h}=\tilde{D} \boldsymbol{u}_{h}^{n+1}$ and $q_{h}=0$ in $(26)$. In particular, for the coupling term $b\left(p_{h}^{n+1}, \tilde{D} \boldsymbol{u}_{h}^{n+1}\right)$, using (26) and (48), we have

$$
\begin{aligned}
b\left(p_{h}^{n+1}, \tilde{D} \boldsymbol{u}_{h}^{n+1}\right) & =j\left(\tilde{D} p_{h}^{n+1}, p_{h}^{n+1}\right) \\
& \geq \frac{1}{4} \bar{D}\left(j\left(p_{h}^{n+1}, p_{h}^{n+1}\right)+j\left(2 p_{h}^{n+1}-p_{h}^{n}, 2 p_{h}^{n+1}-p_{h}^{n}\right)\right),
\end{aligned}
$$

for $2 \leq n \leq N-1$, which corresponds to the BDF2 counterpart of (34). On the other hand for $n=1$, from (26) and (24), we obtain

$$
b\left(p_{h}^{2}, \tilde{D} \boldsymbol{u}_{h}^{2}\right)=\frac{1}{2 \delta t}\left(3 j\left(p_{h}^{2}, p_{h}^{2}\right)-4 j\left(p_{h}^{1}, p_{h}^{2}\right)+b\left(p_{h}^{2}, \boldsymbol{u}_{h}^{0}\right)\right)
$$


If the initial velocity approximation is given in terms of the Ritz-projection, $\boldsymbol{u}_{h}^{0}=P_{h}^{k}\left(\boldsymbol{u}_{0}, 0\right)$, it follows that $b\left(p_{h}^{2}, \boldsymbol{u}_{h}^{0}\right)=j\left(p_{h}^{0}, p_{h}^{2}\right)$, with $p_{h}^{0} \stackrel{\text { def }}{=} R_{h}^{l}\left(\boldsymbol{u}_{0}, 0\right)$. Thus, 50 reduces to

$$
b\left(p_{h}^{2}, \tilde{D} \boldsymbol{u}_{h}^{2}\right)=j\left(\tilde{D} p_{h}^{2}, p_{h}^{2}\right),
$$

so that (49) holds true also for $n=1$.

Finally, if the initial velocity approximation is given in terms of a general interpolant, $\boldsymbol{u}_{h}^{0}=\mathcal{I}_{h}^{k} \boldsymbol{u}_{0}$, we apply an approximation argument (as in (40)). Hence, from 50

$b\left(p_{h}^{2}, \tilde{D} \boldsymbol{u}_{h}^{2}\right) \geq \frac{1}{2 \delta t}\left[3 j\left(p_{h}^{2}, p_{h}^{2}\right)-4 j\left(p_{h}^{1}, p_{h}^{1}\right)-C_{\mathcal{I}}\left(\nu h^{2\left(r_{\boldsymbol{u}}-1\right)}\left\|p_{h}^{2}\right\|_{Q}^{2}+\left\|\boldsymbol{u}_{0}\right\|_{r_{\boldsymbol{u}}, \Omega}^{2}\right)\right]$

which is the BDF2 counterpart of (40) and leads to the stability condition 42. The rest of the proof follows with minor modifications.

Remark 4.7 $A$ bound for the Backward Euler initialization terms $\left\|\boldsymbol{u}_{h}^{1}\right\|_{H}$ and $\left\|\left(\boldsymbol{u}_{h}^{1}, p_{h}^{1}\right)\right\|_{h}$, appearing in the above estimates, is provided by Theorems 4.1 and 4.2 with $n=1$.

Remark 4.8 When the initial velocity approximation is not discretely divergence free, the stability condition (42) has to be satisfied twice when using BDF2: At the first time step (according to (51)) and at the Backward Euler initialization (see Theorem 4.2).

\section{Convergence}

In this paragraph we provide optimal convergence error estimates for the discrete formulation (24), the Backward Euler scheme.

Theorem 5.2 concerns the convergence for the velocity and gives an estimate for the pressure in terms of the error in the velocity time derivative. Theorem 5.3 closes the question of optimal convergence of the pressure by providing an optimal error estimate for the time derivative, provided the exact pressure is smooth. Finally, Theorem 5.4 provides an improved $L^{\infty}((0, T), H)$ estimate that justifies the initialization of the BDF2 scheme with a Backward Euler step.

The following result expresses the modified Galerkin orthogonality in terms of the consistency error in space and time.

Lemma 5.1 (Consistency error) Let $(\boldsymbol{u}, p)$ be the solution of (3) and $\left\{\left(\boldsymbol{u}_{h}^{n}, p_{h}^{n}\right)\right\}_{0 \leq n \leq N}$ the solution of (24). Assume that $\boldsymbol{u} \in \mathcal{C}^{0}([0, T] ; V) \cap \mathcal{C}^{1}((0, T] ; H)$ and $p \in$ $\mathcal{C}^{0}((0, T] ; Q)$. Then, for $0 \leq n \leq N-1$, there holds

$$
\begin{gathered}
\left(\bar{D} \boldsymbol{u}\left(t_{n+1}\right)-\bar{D} \boldsymbol{u}_{h}^{n+1}, \boldsymbol{v}_{h}\right)+a\left(\boldsymbol{u}\left(t_{n+1}\right)-\boldsymbol{u}_{h}^{n+1}, \boldsymbol{v}_{h}\right)+b\left(p\left(t_{n+1}\right)-p_{h}^{n+1}, \boldsymbol{v}_{h}\right) \\
-b\left(q_{h}, \boldsymbol{u}\left(t_{n+1}\right)-\boldsymbol{u}_{h}^{n+1}\right)=j\left(p_{h}^{n+1}, q_{h}\right)+\left(\bar{D} \boldsymbol{u}\left(t_{n+1}\right)-\partial_{t} \boldsymbol{u}\left(t_{n+1}\right), \boldsymbol{v}_{h}\right),
\end{gathered}
$$

for all $\left(\boldsymbol{v}_{h}, q_{h}\right) \in\left[V_{h}^{k}\right]^{d} \times Q_{h}^{l}$.

Theorem 5.2 Assume that $\boldsymbol{u} \in H^{1}\left(0, T ;\left[H^{r}(\Omega)\right]^{d}\right) \cap H^{2}\left(0, T ;\left[L^{2}(\Omega)\right]^{d}\right)$ and $p \in C^{0}\left((0, T] ; H^{s}(\Omega)\right)$ with $r \geq 2$ and $s \geq 1$, and set $\boldsymbol{u}_{h}^{0} \in\left[V_{h}^{k}\right]^{d}$ a given 
approximation of $\boldsymbol{u}_{0}$. Then the following estimate holds for $1 \leq n \leq N$ :

$$
\begin{gathered}
\left\|\boldsymbol{u}_{h}^{n}-\boldsymbol{u}\left(t_{n}\right)\right\|_{H}^{2}+\sum_{m=0}^{n-1} \delta t\left\|\left(\boldsymbol{u}_{h}^{m+1}-\boldsymbol{u}\left(t_{m+1}\right), p_{h}^{m+1}\right)\right\|_{h}^{2} \leq\left\|\mathcal{I}_{h}^{k} \boldsymbol{u}_{0}-\boldsymbol{u}_{h}^{0}\right\|_{H}^{2} \\
+C h^{2 r_{\boldsymbol{u}}}\left(\|\boldsymbol{u}\|_{C^{0}\left(\left[t_{1}, t_{n}\right] ; H^{r} \boldsymbol{u}(\Omega)\right)}^{2}+\nu^{-1}\left\|\partial_{t} \boldsymbol{u}\right\|_{L^{2}\left(0, t_{n} ; H^{\left.r_{u}(\Omega)\right)}\right)}^{2}\right) \\
+C\left(\frac{\delta t^{2}}{\nu}\left\|\partial_{t t} \boldsymbol{u}\right\|_{L^{2}\left(0, t_{n} ; H\right)}^{2}+\frac{h^{2 s_{p}}}{\nu} t_{n}\|p\|_{C^{0}\left(\left[t_{1}, t_{n}\right] ; H^{\left.s_{p}(\Omega)\right)}\right.}^{2}+\nu h^{2\left(r_{\boldsymbol{u}}-1\right)} t_{n}\|\boldsymbol{u}\|_{C^{0}\left(\left[t_{1}, t_{n}\right] ; H^{\left.r_{u}(\Omega)\right)}\right)}^{2}\right) \\
\quad \sum_{m=0}^{n-1} \delta t\left\|p_{h}^{m+1}-p\left(t_{m+1}\right)\right\|_{Q}^{2} \leq C\left(1+\frac{1}{\beta^{2}}\right) \frac{h^{2 s_{p}}}{\nu} t_{n}\|p\|_{C^{0}\left(\left[t_{1}, t_{n}\right] ; H^{s p}(\Omega)\right)}^{2} \\
+\frac{C}{\beta^{2}} \sum_{m=0}^{n-1} \delta t\left(\left\|\left(\boldsymbol{u}_{h}^{m+1}-\boldsymbol{u}\left(t_{m+1}\right), p_{h}^{m+1}\right)\right\|_{h}^{2}+\nu^{-1}\left\|\partial_{t} \boldsymbol{u}\left(t_{m+1}\right)-\bar{D} \boldsymbol{u}_{h}^{m+1}\right\|_{H}^{2}\right),
\end{gathered}
$$

with $C>0$ a positive constant independent of $h$, $\delta t$ and $\nu$.

Proof. The error estimate for the velocity follows standard energy arguments, and for the pressure we use the modified inf-sup condition (13). We start by decomposing the velocity and pressure error using, respectively, the projection $\mathcal{I}_{h}^{k}$ and $\Pi_{h}^{l}$. This yields,

$$
\begin{gathered}
\boldsymbol{u}\left(t_{n+1}\right)-\boldsymbol{u}_{h}^{n+1}=\underbrace{\boldsymbol{u}\left(t_{n+1}\right)-\mathcal{I}_{h}^{k} \boldsymbol{u}\left(t_{n+1}\right)}_{\boldsymbol{\theta}_{\pi}^{n+1}}+\underbrace{\mathcal{I}_{h}^{k} \boldsymbol{u}\left(t_{n+1}\right)-\boldsymbol{u}_{h}^{n+1}}_{\boldsymbol{\theta}_{h}^{n+1}}=\boldsymbol{\theta}_{\pi}^{n+1}+\boldsymbol{\theta}_{h}^{n+1}, \\
p\left(t_{n+1}\right)-p_{h}^{n+1}=\underbrace{p\left(t_{n+1}\right)-\Pi_{h}^{l} p\left(t_{n+1}\right)}_{y_{\pi}^{n+1}}+\underbrace{\prod_{h}^{l} p\left(t_{n+1}\right)-p_{h}^{n+1}}_{y_{h}^{n+1}}=y_{\pi}^{n+1}+y_{h}^{n+1} .
\end{gathered}
$$

The first term $\boldsymbol{\theta}_{\pi}^{n+1}$ can bounded using approximation 10 . In order to estimate $\boldsymbol{\theta}_{h}^{n+1}$ we first note, using 29 and the coercivity of the bi-linear form $a(\cdot, \cdot)+$ $j(\cdot, \cdot)$

$$
\begin{aligned}
& \frac{1}{2} \bar{D}\left\|\boldsymbol{\theta}_{h}^{n+1}\right\|_{H}^{2}+\left\|\left(\boldsymbol{\theta}_{h}^{n+1}, y_{h}^{n+1}\right)\right\|_{h}^{2} \leq\left(\bar{D} \boldsymbol{\theta}_{h}^{n+1}, \boldsymbol{\theta}_{h}^{n+1}\right)+\left\|\left(\boldsymbol{\theta}_{h}^{n+1}, y_{h}^{n+1}\right)\right\|_{h}^{2} \\
\leq & \underbrace{\left(\bar{D} \boldsymbol{\theta}_{h}^{n+1}, \boldsymbol{\theta}_{h}^{n+1}\right)+a\left(\boldsymbol{\theta}_{h}^{n+1}, \boldsymbol{\theta}_{h}^{n+1}\right)+b\left(y_{h}^{n+1}, \boldsymbol{\theta}_{h}^{n+1}\right)-b\left(y_{h}^{n+1}, \boldsymbol{\theta}_{h}^{n+1}\right)+j\left(y_{h}^{n+1}, y_{h}^{n+1}\right)}_{T_{1}^{n+1}} .
\end{aligned}
$$

In addition, using $(52)$ we have

$$
\begin{aligned}
T_{1}^{n+1}= & -\left(\bar{D} \boldsymbol{\theta}_{\pi}^{n+1}, \boldsymbol{\theta}_{h}^{n+1}\right)-a\left(\boldsymbol{\theta}_{\pi}^{n+1}, \boldsymbol{\theta}_{h}^{n+1}\right)+j\left(\Pi_{h}^{l} p\left(t_{n+1}\right), y_{h}^{n+1}\right)-b\left(y_{\pi}^{n+1}, \boldsymbol{\theta}_{h}^{n+1}\right) \\
& +b\left(y_{h}^{n+1}, \boldsymbol{\theta}_{\pi}^{n+1}\right)+\left(\bar{D} \boldsymbol{u}\left(t_{n+1}\right)-\bar{D} \boldsymbol{u}_{h}^{n+1}, \boldsymbol{\theta}_{h}^{n+1}\right)+a\left(\boldsymbol{u}\left(t_{n+1}\right)-\boldsymbol{u}_{h}^{n+1}, \boldsymbol{\theta}_{h}^{n+1}\right) \\
& +b\left(p\left(t_{n+1}\right)-p_{h}^{n+1}, \boldsymbol{\theta}_{h}^{n+1}\right)-b\left(y_{h}^{n+1}, \boldsymbol{u}\left(t_{n+1}\right)-\boldsymbol{u}_{h}^{n+1}\right)-j\left(p_{h}^{n+1}, y_{h}^{n+1}\right) .
\end{aligned}
$$

By the modified Galerkin orthogonality (Lemma 5.1) this expression reduces to

$$
\begin{aligned}
T_{1}^{n+1}= & -\left(\bar{D} \boldsymbol{\theta}_{\pi}^{n+1}, \boldsymbol{\theta}_{h}^{n+1}\right)+\left(\bar{D} \boldsymbol{u}\left(t_{n+1}\right)-\partial_{t} \boldsymbol{u}\left(t_{n+1}\right), \boldsymbol{\theta}_{h}^{n+1}\right) \\
& -a\left(\boldsymbol{\theta}_{\pi}^{n+1}, \boldsymbol{\theta}_{h}^{n+1}\right)+j\left(\Pi_{h}^{l} p\left(t_{n+1}\right), y_{h}^{n+1}\right)-b\left(y_{\pi}^{n+1}, \boldsymbol{\theta}^{n+1}\right)+b\left(y_{h}^{n+1}, \boldsymbol{\theta}_{\pi}^{n+1}\right) .
\end{aligned}
$$


Now, using the Cauchy-Schwarz and the Poincaré's inequalities and (11), we have

$$
\begin{gathered}
T_{1}^{n+1} \leq \underbrace{\left(\left\|\bar{D} \boldsymbol{u}\left(t_{n+1}\right)-\partial_{t} \boldsymbol{u}\left(t_{n+1}\right)\right\|_{H}+\left\|\bar{D} \boldsymbol{\theta}_{\pi}^{n+1}\right\|_{H}\right)}_{T_{2}^{n+1}} \frac{C_{\mathrm{p}}}{\nu^{\frac{1}{2}}}\left\|\left(\boldsymbol{\theta}_{h}^{n+1}, y_{h}^{n+1}\right)\right\| \\
+\left(\left\|\boldsymbol{\theta}_{\pi}^{n+1}\right\|_{V}+\left\|y_{\pi}^{n+1}\right\|_{Q}+\nu^{\frac{1}{2}}\left\|h^{-1} \boldsymbol{\theta}_{\pi}^{n+1}\right\|_{H}+j\left(\Pi_{h}^{l} p\left(t_{n+1}\right), \Pi_{h}^{l} p\left(t_{n+1}\right)\right)^{\frac{1}{2}}\right)\left\|\left(\boldsymbol{\theta}_{h}^{n+1}, y_{h}^{n+1}\right)\right\| .
\end{gathered}
$$

The term $T_{2}^{n+1}$ can be treated, in a standard way (see e.g 30 ), using a Taylor expansion and the Cauchy-Schwarz inequality, which yields

$$
\begin{aligned}
T_{2}^{n+1} & \leq \frac{1}{\delta t} \int_{t_{n}}^{t_{n+1}}\left(\delta t\left\|\partial_{t t} \boldsymbol{u}(s)\right\|_{H}+\left\|\partial_{t} \boldsymbol{\theta}_{\pi}(s)\right\|_{H}\right) \mathrm{d} s \\
& \leq \delta t^{\frac{1}{2}}\left\|\partial_{t t} \boldsymbol{u}(s)\right\|_{L^{2}\left(\left(t_{n}, t_{n+1}\right) ; H\right)}+\delta t^{-\frac{1}{2}}\left\|\partial_{t} \boldsymbol{\theta}_{\pi}\right\|_{L^{2}\left(\left(t_{n}, t_{n+1}\right) ; H\right)}
\end{aligned}
$$

Thus, from 55, using Young's inequality, it follows that

$$
\begin{aligned}
T_{1}^{n+1} & \leq \frac{1}{2}\left\|\left(\boldsymbol{\theta}_{h}^{n+1}, y_{h}^{n+1}\right)\right\|^{2}+C\left[\frac{C_{\mathrm{P}}^{2}}{\nu}\left(\delta t\left\|\partial_{t t} \boldsymbol{u}\right\|_{L^{2}\left(\left(t_{n}, t_{n+1}\right) ; H\right)}^{2}+\delta t^{-1}\left\|\partial_{t} \boldsymbol{\theta}_{\pi}\right\|_{L^{2}\left(\left(t_{n}, t_{n+1}\right) ; H\right)}^{2}\right)\right. \\
& \left.+\left\|\boldsymbol{\theta}_{\pi}^{n+1}\right\|_{V}^{2}+\left\|y_{\pi}^{n+1}\right\|_{Q}^{2}+\nu\left\|h^{-1} \boldsymbol{\theta}_{\pi}^{n+1}\right\|_{H}^{2}+j\left(\Pi_{h}^{l} p\left(t_{n+1}\right), \Pi_{h}^{l} p\left(t_{n+1}\right)\right)\right]
\end{aligned}
$$

By inserting this expression in $(53)$, multiplying the resulting expression by $2 \delta t$ and after summation over $0 \leq m \leq n-1$ we obtain

$$
\begin{aligned}
& \left\|\boldsymbol{\theta}_{h}^{n}\right\|_{H}^{2}+\sum_{m=0}^{n-1} \delta t\left\|\left(\boldsymbol{\theta}_{h}^{m+1}, y_{h}^{m+1}\right)\right\|_{h}^{2} \leq\left\|\boldsymbol{\theta}_{h}^{0}\right\|_{H}^{2}+C\left[\delta t^{2} \nu^{-1}\left\|\partial_{t t} \boldsymbol{u}\right\|_{L^{2}\left(0, t_{n} ; H\right)}^{2}+\nu^{-1}\left\|\partial_{t} \boldsymbol{\theta}_{\pi}\right\|_{L^{2}\left(0, t_{n} ; H\right)}^{2}\right. \\
+ & \left.\sum_{m=0}^{n-1} \delta t\left(\left\|\boldsymbol{\theta}_{\pi}^{m+1}\right\|_{V}^{2}+\left\|y_{\pi}^{m+1}\right\|_{Q}^{2}+\nu\left\|h^{-1} \boldsymbol{\theta}_{\pi}^{m+1}\right\|_{H}^{2}+j\left(\Pi_{h}^{l} p\left(t_{m+1}\right), \Pi_{h}^{l} p\left(t_{m+1}\right)\right)\right)\right] .
\end{aligned}
$$

Finally, the velocity error estimate is obtained using approximation 10 and the consistency of the pressure stabilization (8), which yields

$$
\begin{aligned}
\left\|\boldsymbol{\theta}_{h}^{n}\right\|_{H}^{2}+\sum_{m=0}^{n-1} \delta t\left\|\left(\boldsymbol{\theta}_{h}^{m+1}, y_{h}^{m+1}\right)\right\|_{h}^{2} \leq\left\|\boldsymbol{\theta}_{h}^{0}\right\|_{H}^{2} \\
\quad+C\left[\frac{\delta t^{2}}{\nu}\left\|\partial_{t t} \boldsymbol{u}\right\|_{L^{2}\left(0, t_{n} ; H\right)}^{2}+\frac{h^{2 r_{\boldsymbol{u}}}}{\nu}\left\|\partial_{t} \boldsymbol{u}\right\|_{L^{2}\left(0, t_{n} ; H^{\left.r_{u}(\Omega)\right)}\right.}^{2}\right. \\
\left.\quad+\nu h^{2\left(r_{\boldsymbol{u}}-1\right)} \sum_{m=0}^{n-1} \delta t\left\|\boldsymbol{u}\left(t_{m+1}\right)\right\|_{r_{\boldsymbol{u}}, \Omega}^{2}+\frac{h^{2 s_{p}}}{\nu} \sum_{m=0}^{n-1} \delta t\left\|p\left(t_{m+1}\right)\right\|_{s_{p}, \Omega}^{2}\right],
\end{aligned}
$$

For the pressure error estimate we first note that, from $\sqrt[52]{ }$, it suffices to control $\left\|y_{h}^{n+1}\right\|_{0, \Omega}$. To this aim, we use the modified inf-sup condition (13):

$$
\beta\left\|y_{h}^{n+1}\right\|_{Q} \leq \sup _{\boldsymbol{v}_{h} \in\left[V_{h}^{k}\right]^{d}} \frac{\left|b\left(y_{h}^{n+1}, \boldsymbol{v}_{h}\right)\right|}{\left\|\boldsymbol{v}_{h}\right\|_{V}}+C j\left(y_{h}^{n+1}, y_{h}^{n+1}\right)^{\frac{1}{2}} .
$$


From 52 we get

$$
b\left(y_{h}^{n+1}, \boldsymbol{v}_{h}\right)=-b\left(y_{\pi}^{n+1}, \boldsymbol{v}_{h}\right)+b\left(p\left(t_{n+1}\right)-p_{h}^{n+1}, \boldsymbol{v}_{h}\right) .
$$

The first term can be bounded, using the continuity of $b(\cdot, \cdot),(5)$, this yields

$$
b\left(y_{\pi}^{n+1}, \boldsymbol{v}_{h}\right) \leq\left\|y_{\pi}^{n+1}\right\|_{Q}\left\|\boldsymbol{v}_{h}\right\|_{V} .
$$

On the other hand, using the modified Galerkin orthogonality (Lemma 5.1 with $\left.q_{h}=0\right)$ we have

$$
\begin{aligned}
b\left(p\left(t_{n+1}\right)-p_{h}^{n+1}, \boldsymbol{v}_{h}\right) & =-a\left(\boldsymbol{u}\left(t_{n+1}\right)-\boldsymbol{u}_{h}^{n+1}, \boldsymbol{v}_{h}\right)-\left(\partial_{t} \boldsymbol{u}\left(t_{n+1}\right)-\bar{D} \boldsymbol{u}_{h}^{n+1}, \boldsymbol{v}_{h}\right) \\
& \leq C\left\|\left(\boldsymbol{u}\left(t_{n+1}\right)-\boldsymbol{u}_{h}^{n+1}, 0\right)\right\|_{h}\left\|\boldsymbol{v}_{h}\right\|_{V}+\left\|\partial_{t} \boldsymbol{u}\left(t_{n+1}\right)-\bar{D} \boldsymbol{u}_{h}^{n+1}\right\|_{H}\left\|\boldsymbol{v}_{h}\right\|_{H}
\end{aligned}
$$

As a result, from the above estimations we have:

$$
\beta\left\|y_{h}^{n+1}\right\|_{Q} \leq C\left(\left\|y_{\pi}^{n+1}\right\|_{Q}+\left\|\left(\boldsymbol{u}\left(t_{n+1}\right)-\boldsymbol{u}_{h}^{n+1}, y_{h}^{n+1}\right)\right\|_{h}\right)+\frac{C_{P}}{\nu^{\frac{1}{2}}}\left\|\partial_{t} \boldsymbol{u}\left(t_{n+1}\right)-\bar{D} \boldsymbol{u}_{h}^{n+1}\right\|_{H} .
$$

Therefore,

$$
\begin{aligned}
\beta^{2} \sum_{m=0}^{n-1} \delta t\left\|y_{h}^{m+1}\right\|_{Q}^{2} \leq & C \sum_{m=0}^{n-1} \delta t\left(\left\|y_{\pi}^{m+1}\right\|_{Q}^{2}+\left\|\left(\boldsymbol{u}\left(t_{m+1}\right)-\boldsymbol{u}_{h}^{m+1}, y_{h}^{m+1}\right)\right\|_{h}^{2}\right. \\
& \left.+\nu^{-1}\left\|\partial_{t} \boldsymbol{u}\left(t_{m+1}\right)-\bar{D} \boldsymbol{u}_{h}^{m+1}\right\|_{H}^{2}\right)
\end{aligned}
$$

and we conclude using approximation and the error estimate for the velocity. $\square$

We close the problem of the pressure convergence by providing an error estimate for the time derivative of the velocity.

Theorem 5.3 Under the assumptions of Theorem 5.2, assuming that $p \in C^{0}\left([0, T] ; H^{s}(\Omega)\right)$, $\boldsymbol{u}_{0} \in V \cap H_{0}(\operatorname{div} ; \Omega)$ and if $\boldsymbol{u}_{h}^{0} \stackrel{\text { def }}{=} P_{h}^{k}\left(\boldsymbol{u}_{0}, 0\right)$, for $1 \leq n \leq N$, we have

$$
\begin{aligned}
& \sum_{m=0}^{n-1} \delta t\left\|\bar{D} \boldsymbol{u}_{h}^{m+1}-\partial_{t} \boldsymbol{u}\left(t_{m+1}\right)\right\|_{H}^{2}+\left\|\left(P_{h}^{k}\left(\boldsymbol{u}\left(t_{n}\right), p\left(t_{n}\right)\right)-\boldsymbol{u}_{h}^{n}, R_{h}^{l}\left(\boldsymbol{u}\left(t_{n}\right), p\left(t_{n}\right)\right)-p_{h}^{n}\right)\right\|_{h}^{2} \\
& \quad \leq C\left(\delta t^{2}\left\|\partial_{t t} \boldsymbol{u}\right\|_{L^{2}(0, T ; H)}^{2}+h^{2 r_{u}}\left\|\partial_{t} \boldsymbol{u}\right\|_{L^{2}\left(0, T ; H^{r} \boldsymbol{u}(\Omega)\right)}^{2}\right)+C \frac{h^{2 s_{p}}}{\nu}\|p(0)\|_{s_{p}, \Omega}^{2} .
\end{aligned}
$$

Proof. In order to provide an optimal error estimate, we decompose the error in terms of the Ritz-projection operator 21

$$
\begin{aligned}
\boldsymbol{u}\left(t_{n+1}\right)-\boldsymbol{u}_{h}^{n+1} & =\underbrace{\boldsymbol{u}\left(t_{n+1}\right)-P_{h}^{k}\left(\boldsymbol{u}\left(t_{n+1}\right), p\left(t_{n+1}\right)\right)}_{\boldsymbol{\theta}_{\pi}^{n+1}}+\underbrace{P_{h}^{k}\left(\boldsymbol{u}\left(t_{n+1}\right), p\left(t_{n+1}\right)\right)-\boldsymbol{u}_{h}^{n+1}}_{\boldsymbol{\theta}_{h}^{n+1}} \\
& =\boldsymbol{\theta}_{\pi}^{n+1}+\boldsymbol{\theta}_{h}^{n+1}, \\
p\left(t_{n+1}\right)-p_{h}^{n+1} & =\underbrace{p\left(t_{n+1}\right)-R_{h}^{l}\left(\boldsymbol{u}\left(t_{n+1}\right), p\left(t_{n+1}\right)\right)}_{y_{\pi}^{n+1}}+\underbrace{R_{h}^{l}\left(\boldsymbol{u}\left(t_{n+1}\right), p\left(t_{n+1}\right)\right)-p_{h}^{n+1}}_{y_{h}^{n+1}} \\
& =y_{\pi}^{n+1}+y_{h}^{n+1} .
\end{aligned}
$$


Using the triangle inequality we then have

$$
\begin{aligned}
& \sum_{m=0}^{n-1} \delta t\left\|\partial_{t} \boldsymbol{u}\left(t_{m+1}\right)-\bar{D} \boldsymbol{u}_{h}^{m+1}\right\|_{H}^{2} \\
& \leq C \sum_{m=0}^{n-1} \delta t\left(\left\|\partial_{t} \boldsymbol{u}\left(t_{m+1}\right)-\bar{D} \boldsymbol{u}\left(t_{m+1}\right)\right\|_{H}^{2}+\left\|\bar{D} \boldsymbol{\theta}_{\pi}^{m+1}\right\|_{H}^{2}+\left\|\bar{D} \boldsymbol{\theta}_{h}^{m+1}\right\|_{H}^{2}\right) .
\end{aligned}
$$

For the first term we proceed as in 56 using a Taylor expansion, which yields

$$
\left\|\partial_{t} \boldsymbol{u}\left(t_{n+1}\right)-\bar{D} \boldsymbol{u}\left(t_{n+1}\right)\right\|_{H}^{2} \leq \delta t^{\frac{1}{2}}\left\|\partial_{t t} \boldsymbol{u}(s)\right\|_{L^{2}\left(\left(t_{n}, t_{n+1}\right) ; H\right)} .
$$

For the second term we have,

$$
\left\|\bar{D} \boldsymbol{\theta}_{\pi}^{n+1}\right\|_{H}=\frac{1}{\delta t} \int_{t_{n}}^{t_{n+1}}\left\|\partial_{t} \boldsymbol{\theta}_{\pi}(s)\right\|_{H} \mathrm{~d} s \leq \delta t^{-\frac{1}{2}}\left\|\partial_{t} \boldsymbol{\theta}_{\pi}\right\|_{L^{2}\left(\left(t_{n}, t_{n+1}\right) ; H\right)}
$$

Finally, for the third term we use the modified Galerkin orthogonality (Lemma 5.1 with $q_{h}=0$ ) and the definition of the Ritz-projection (21) to obtain

$$
\begin{aligned}
&\left\|\bar{D} \boldsymbol{\theta}_{h}^{n+1}\right\|_{H}^{2}+a\left(\boldsymbol{\theta}_{h}^{n+1}, \bar{D} \boldsymbol{\theta}_{h}^{n+1}\right)+b\left(y_{h}^{n+1}, \bar{D} \boldsymbol{\theta}_{h}^{n+1}\right) \\
&=-\left(\bar{D} \boldsymbol{\theta}_{\pi}^{n+1}, \bar{D} \boldsymbol{\theta}_{h}^{n+1}\right)-a\left(\boldsymbol{\theta}_{\pi}^{n+1}, \bar{D} \boldsymbol{\theta}_{h}^{n+1}\right)-b\left(y_{\pi}^{n+1}, \bar{D} \boldsymbol{\theta}_{h}^{n+1}\right)+\left(\bar{D} \boldsymbol{u}\left(t_{n+1}\right)-\partial_{t} \boldsymbol{u}\left(t_{n+1}\right), \bar{D} \boldsymbol{\theta}_{h}^{n+1}\right) \\
&=-\left(\bar{D} \boldsymbol{\theta}_{\pi}^{n+1}, \bar{D} \boldsymbol{\theta}_{h}^{n+1}\right)+\left(\bar{D} \boldsymbol{u}\left(t_{n+1}\right)-\partial_{t} \boldsymbol{u}\left(t_{n+1}\right), \bar{D} \boldsymbol{\theta}_{h}^{n+1}\right) .
\end{aligned}
$$

Young's inequality yields:

$$
\begin{aligned}
& \frac{1}{2}\left\|\bar{D} \boldsymbol{\theta}_{h}^{n+1}\right\|_{H}^{2}+a\left(\boldsymbol{\theta}_{h}^{n+1}, \bar{D} \boldsymbol{\theta}_{h}^{n+1}\right)+b\left(y_{h}^{n+1}, \bar{D} \boldsymbol{\theta}_{h}^{n+1}\right) \\
& \leq C\left(\left\|\bar{D} \boldsymbol{\theta}_{\pi}^{n+1}\right\|_{H}^{2}+\left\|\bar{D} \boldsymbol{u}\left(t_{n+1}\right)-\partial_{t} \boldsymbol{u}\left(t_{n+1}\right)\right\|_{H}^{2}\right) .
\end{aligned}
$$

In addition, for $0 \leq n \leq N$, testing (21) at the time level $n$ with $\boldsymbol{v}_{h}=\mathbf{0}$ we have

$$
b\left(q_{h}, P_{h}^{k}\left(\boldsymbol{u}\left(t_{n}\right), p\left(t_{n}\right)\right)\right)=j\left(R_{h}^{l}\left(\boldsymbol{u}\left(t_{n}\right), p\left(t_{n}\right)\right), q_{h}\right) .
$$

On the other hand, for $1 \leq n \leq N$, testing (24) at the time level $n$ with $\boldsymbol{v}_{h}=\mathbf{0}$ and since, by definition, $\boldsymbol{u}_{h}^{0} \stackrel{\text { def }}{=} P_{h}^{k}\left(\boldsymbol{u}_{0}, 0\right)$ we have

$$
b\left(q_{h}, \boldsymbol{u}_{h}^{n}\right)=j\left(p_{h}^{n}, q_{h}\right),
$$

for all $q_{h} \in Q_{h}^{l}$ and $0 \leq n \leq N$ and where we have defined $p_{h}^{0} \stackrel{\text { def }}{=} R_{h}^{l}\left(\boldsymbol{u}_{0}, 0\right)$. As a result, from (61)- 62 , we have

$$
b\left(q_{h}, \boldsymbol{\theta}_{h}^{n}\right)=j\left(y_{h}^{n}, q_{h}\right),
$$

for all $q_{h} \in Q_{h}^{l}$ and $0 \leq n \leq N$. We therefore have, for $0 \leq n \leq N-1$

$$
b\left(y_{h}^{n+1}, \bar{D} \boldsymbol{\theta}_{h}^{n+1}\right)=j\left(\bar{D} y_{h}^{n+1}, y_{h}^{n+1}\right) .
$$

On the other hand, using the symmetry of $a$ and $j$, we have

$$
\begin{aligned}
a\left(\boldsymbol{\theta}_{h}^{n+1}, \bar{D} \boldsymbol{\theta}_{h}^{n+1}\right) & =\frac{1}{2} \bar{D} a\left(\boldsymbol{\theta}_{h}^{n+1}, \boldsymbol{\theta}_{h}^{n+1}\right)+\frac{\delta t}{2} a\left(\bar{D} \boldsymbol{\theta}_{h}^{n+1}, \bar{D} \boldsymbol{\theta}_{h}^{n+1}\right), \\
j\left(y_{h}^{n+1}, \bar{D} y_{h}^{n+1}\right) & =\frac{1}{2} \bar{D} j\left(y_{h}^{n+1}, y_{h}^{n+1}\right)+\frac{\delta t}{2} j\left(\bar{D} y_{h}^{n+1}, \bar{D} y_{h}^{n+1}\right) .
\end{aligned}
$$


So that

$$
\begin{aligned}
\frac{1}{2}\left\|\bar{D} \boldsymbol{\theta}_{h}^{n+1}\right\|_{H}^{2}+\frac{1}{2} \bar{D}\left(a\left(\boldsymbol{\theta}_{h}^{n+1}, \boldsymbol{\theta}_{h}^{n+1}\right)+j\left(y_{h}^{n+1}, y_{h}^{n+1}\right)\right) \\
\leq\left\|\bar{D} \boldsymbol{\theta}_{\pi}^{n+1}\right\|_{H}^{2}+\left\|\bar{D} \boldsymbol{u}\left(t_{n+1}\right)-\partial_{t} \boldsymbol{u}\left(t_{n+1}\right)\right\|_{H}^{2} .
\end{aligned}
$$

So that, after multiplication by $2 \delta t$ and summation over $0 \leq n \leq N-1$ we have

$$
\begin{aligned}
\sum_{m=0}^{n-1} \delta t\left\|\bar{D} \boldsymbol{\theta}_{h}^{m+1}\right\|_{H}^{2} & +\left\|\left(\boldsymbol{\theta}_{h}^{n}, y_{h}^{n}\right)\right\|_{h}^{2} \leq\left\|\left(\boldsymbol{\theta}_{h}^{0}, y_{h}^{0}\right)\right\|_{h}^{2} \\
& +C \sum_{m=0}^{n-1} \delta t\left(\left\|\bar{D} \boldsymbol{\theta}_{\pi}^{m+1}\right\|_{H}^{2}+\left\|\bar{D} \boldsymbol{u}\left(t_{m+1}\right)-\partial_{t} \boldsymbol{u}\left(t_{m+1}\right)\right\|_{H}^{2}\right) .
\end{aligned}
$$

For the initial terms we use the linearity of the Ritz-projection and its approximation properties (Lemma 3.4) to obtain

$$
\begin{aligned}
\left\|\left(\boldsymbol{\theta}_{h}^{0}, y_{h}^{0}\right)\right\|_{h}^{2} & =\left\|\left(P_{h}^{k}(\mathbf{0}, p(0)), R_{h}^{l}(\mathbf{0}, p(0))\right)\right\|_{h}^{2} \\
& \leq \frac{C}{\nu} h^{2 s_{p}}\|p(0)\|_{s_{p}, \Omega}^{2} .
\end{aligned}
$$

Therefore, using 60 and $(56)$, we have

$$
\sum_{m=0}^{n-1} \delta t\left\|\bar{D} \boldsymbol{\theta}_{h}^{m+1}\right\|_{H}^{2}+\left\|\left(\boldsymbol{\theta}_{h}^{n}, y_{h}^{n}\right)\right\|_{h}^{2} \leq C\left(\frac{h^{2 s_{p}}}{\nu}\|p(0)\|_{s_{p}, \Omega}^{2}+\delta t^{2}\left\|\partial_{t t} \boldsymbol{u}\right\|_{L^{2}\left(0, t_{n} ; H\right)}^{2}\right),
$$

for $1 \leq n \leq N$.

Finally, for completeness, we here give a result of optimal convergence in the $L^{\infty}((0, T), H)$-norm. For this we assume that the domain $\Omega$ is such that the optimal convergence in the $H$-norm holds for the Ritz-projection (see Lemma 3.4. This result is of importance since it shows that the initialization of the BDF2 method using one BDF1 step is justified (i.e. we keep error optimality in time).

Theorem 5.4 Assume that the domain $\Omega$ is sufficiently smooth so that the $H$ estimate (23) holds. Assume also that $\boldsymbol{u} \in H^{1}\left(0, T ;\left[H_{\boldsymbol{u}}^{r}(\Omega)\right]^{d}\right) \cap H^{2}\left(0, T ;\left[L^{2}(\Omega)\right]^{d}\right)$, $p \in C^{0}\left([0, T] ; H^{s_{p}}(\Omega)\right)$ with $r_{\boldsymbol{u}} \geq 2, s_{p} \geq 1, \boldsymbol{u}_{0} \in V \cap H_{0}(\operatorname{div} ; \Omega)$ and $\boldsymbol{u}_{h}^{0} \stackrel{\text { def }}{=}$ $P_{h}^{k}\left(\boldsymbol{u}_{0}, 0\right)$. Then the following estimate holds for $1 \leq n \leq N$ :

$$
\begin{aligned}
\left\|\boldsymbol{u}\left(t_{n}\right)-\boldsymbol{u}_{h}^{n}\right\|_{H} \leq \frac{C}{\nu^{\frac{1}{2}}}\left(h^{r_{\boldsymbol{u}}}\right. & \left\|\boldsymbol{u}_{0}\right\|_{r_{\boldsymbol{u}}, \Omega}+h^{s_{p}+1}\|p(0)\|_{s_{p}, \Omega} \\
& \left.\quad+h^{r_{u}}\left\|\partial_{t} \boldsymbol{u}\right\|_{L^{1}\left(0, t_{n} ; H^{\left.r_{u}(\Omega)\right)}\right.}+\delta t\left\|\partial_{t t} \boldsymbol{u}\right\|_{L^{1}\left(0, t_{n} ; H\right)}\right) .
\end{aligned}
$$

Proof. Since the proof is similar to that of Theorem 5.3 and we will only give the outline. Let $\boldsymbol{\theta}_{h}^{n+1}$ and $y_{h}^{n+1}$ be defined as in (58). From (53) and (54), it follows that

$$
\left(\bar{D} \boldsymbol{\theta}_{h}^{n+1}, \boldsymbol{\theta}_{h}^{n+1}\right) \leq-\left(\bar{D} \boldsymbol{\theta}_{\pi}^{n+1}, \boldsymbol{\theta}_{h}^{n+1}\right)+\left(\bar{D} \boldsymbol{u}\left(t_{n+1}\right)-\partial_{t} \boldsymbol{u}\left(t_{n+1}\right), \boldsymbol{\theta}_{h}^{n+1}\right) .
$$


Applying now the Cauchy-Schwarz inequality, we have

$$
\left\|\boldsymbol{\theta}_{h}^{n+1}\right\|_{H} \leq\left\|\boldsymbol{\theta}_{h}^{n}\right\|_{H}+\delta t\left(\left\|\bar{D} \boldsymbol{\theta}_{\pi}^{n+1}\right\|_{H}+\left\|\bar{D} \boldsymbol{u}\left(t_{n+1}\right)-\partial_{t} \boldsymbol{u}\left(t_{n+1}\right)\right\|_{H}\right),
$$

and by summation over $n$, we get

$$
\left\|\boldsymbol{\theta}_{h}^{n}\right\|_{H} \leq\left\|\boldsymbol{\theta}_{h}^{0}\right\|_{H}+\sum_{m=0}^{n-1} \delta t\left(\left\|\bar{D} \boldsymbol{\theta}_{\pi}^{n+1}\right\|_{H}+\left\|\bar{D} \boldsymbol{u}\left(t_{n+1}\right)-\partial_{t} \boldsymbol{u}\left(t_{n+1}\right)\right\|_{H}\right),
$$

for $1 \leq n \leq N$. The first term in the right hand side can be estimated using Lemma 3.4 since, by definition,

$$
\boldsymbol{\theta}_{h}^{0}=P_{h}^{k}(\boldsymbol{u}(0), p(0))-\boldsymbol{u}_{h}^{0}=P_{h}^{k}\left(\boldsymbol{u}_{0}, p(0)\right)-P_{h}^{k}\left(\boldsymbol{u}_{0}, 0\right)=P_{h}^{k}(\mathbf{0}, p(0)) .
$$

Finally, for the finite difference consistency terms we use a standard argument (see e.g. [34, Theorem 1.5, Page 14]).

Remark 5.5 From (64), one could pretend to initialize the time-stepping procedure with $\boldsymbol{u}_{h}^{0}=P_{h}^{k}\left(\boldsymbol{u}_{0}, p(0)\right)$ (as in [33], for instance). In practice, however, the initial pressure is unknown, so that the choice $\boldsymbol{u}_{h}^{0}=P_{h}^{k}\left(\boldsymbol{u}_{0}, 0\right)$ is more convenient. Lemma 3.4 shows that we can preserve optimality while keeping this choice (see also [4]).

Remark 5.6 Note that the above convergence proofs only use stability, Galerkin orthogonality and the truncation error of the finite difference time approximation scheme. Hence the extension to the second order Crank-Nicholson or BDF2 schemes is straightforward. In particular we recall that the estimate of Lemma 5.4 shows that the initialization using one BDF1 step does not make the convergence deteriorate, provided the solution is sufficiently smooth under the first timestep. Indeed, for smooth solutions we expect $\left\|\partial_{t t} \boldsymbol{u}\right\|_{L^{1}(0, \delta t ; H)}$ to be $O(\delta t)$ and hence the global convergence will be second order in spite of the intial low order perturbation.

\section{$6 \quad$ Numerical experiments}

In this section we will consider some numerical examples using the continuous interior penalty stabilization (CIP), described in \$3.1.1. We present computations demonstrating the optimal convergence using finite element spaces consisting of quadratic functions, for the space discretization, and first and second order backward difference formulas and the Crank-Nicholson scheme for the time discretization. We also verify numerically that, for small time steps, the pressure is unstable for initial data that are not discretely divergence free. All computations have been performed using FreeFem $++[26]$.

\subsection{Convergence rate in time}

We consider problem (3) in two dimensions, $\Omega=[0,1] \times[0,1]$ and $T=1$, with non-homogeneous boundary conditions. The right hand side $f$ and the 
boundary and initial data are chosen in order to ensure that the exact solution is given by:

$$
\begin{aligned}
& \boldsymbol{u}(x, y, t)=g(t)\left(\begin{array}{c}
\sin (\pi x-0.7) \sin (\pi y+0.2) \\
\cos (\pi x-0.7) \cos (\pi y+0.2)
\end{array}\right), \\
& p(x, y, t)=g(t)(\sin (x) \cos (y)+(\cos (1)-1) \sin (1)),
\end{aligned}
$$

with $g(t)=1+t^{5}+e^{-\frac{t}{10}}+\sin (t)$.

In order to illustrate the convergence rate in time of the discrete solution, we have used quadratic approximations in space and a mesh parameter $h=0.01$. In this case, the stability condition 42 is always satisfied for the range of time steps considered. Thus, the choice of the Lagrange interpolant or of the Ritz-projection as approximation of the initial velocity give similar results.

In figures 1(a)-(c) we report the convergences of the errors for the velocities $\left(\|\cdot\|_{L^{\infty}\left(0, T ; L^{2}(\Omega)\right)}\right)$ and the pressures $\left(\|\cdot\|_{L^{2}\left(0, T ; L^{2}(\Omega)\right)}\right)$ for the first order backward difference formula (BDF1), Crank-Nicholson and the second order backward difference formulas (BDF2). In all the numerical examples both the velocities and the pressures converge at the optimal rate $(O(\delta t)$ for $\mathrm{BDF} 1$ and $O\left(\delta t^{2}\right)$ for Crank-Nicholson and BDF2). The BDF2 scheme was initialized using one step of BDF1.

\subsection{Behavior in the small time step limit}

In this paragraph we illustrate the impact of the initial velocity approximation on the approximate pressures for small time steps. For non discrete divergence free initial approximations, a pressure instability is predicted by Corollary 4.3 unless condition 42 is satisfied. In other words, pressure instabilities are expected for very small time steps.

We consider problem (3) in two dimensions and with non-homogeneous boundary conditions. We set $\Omega=[0,1] \times[0,1]$, and the right hand side $\boldsymbol{f}$ and the boundary data are chosen in order to ensure that the exact (steady) solution is given by:

$$
\begin{aligned}
& \boldsymbol{u}(x, y, t)=\left(\begin{array}{c}
\sin (\pi x-0.7) \sin (\pi y+0.2) \\
\cos (\pi x-0.7) \cos (\pi y+0.2)
\end{array}\right) \\
& p(x, y, t)=\sin x \cos y+(\cos (1)-1) \sin (1) .
\end{aligned}
$$

This numerical experiment is, in some degree, motivated by the work reported in [3] (see also [19]), where pressure instabilities, of a different nature, are illustrated for pressure stabilizations involving residuals of the partial differential equation (e.g PSPG and GaLS). Indeed, the time derivative involved in the residual perturbs the coercivity of the space semi-discrete operator, which leads to pressure instabilities for (sufficiently) small time steps (see [3]). Let us emphasize that, according to section $\$ 4$, such kind of instabilities do not appear here, in particular since the CIP pressure stabilization (and the other examples of paragraph $\$ 3.1 .1$ is consistent without introducing the time derivative.

For different initial velocity approximations, we compare the behavior of the error in the pressure after one time step of the Backward Euler scheme, i.e.,

$$
\delta t^{\frac{1}{2}}\left\|p\left(t_{1}\right)-p_{h}^{1}\right\|_{Q} .
$$




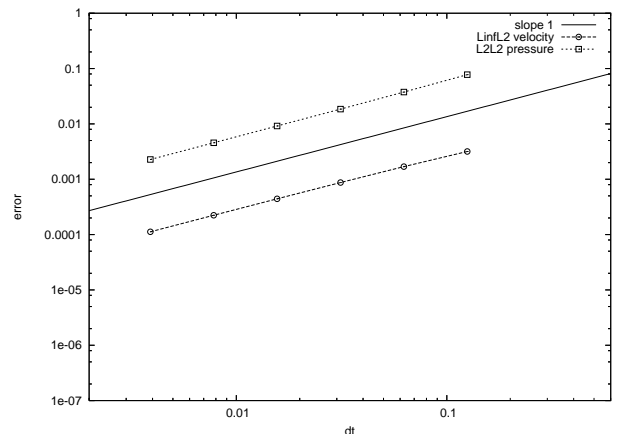

(a) Backward Euler (BDF1) scheme

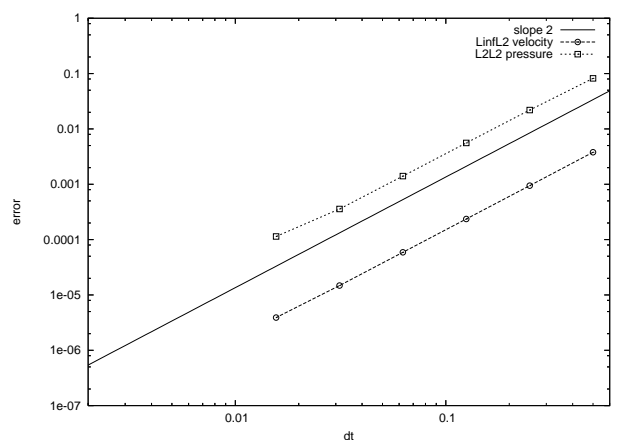

(b) Crank-Nicholson

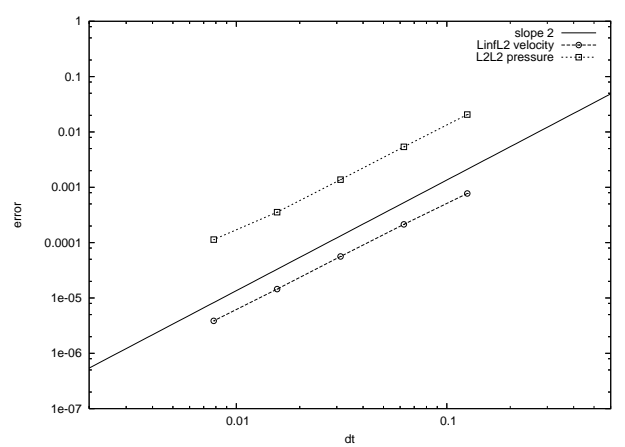

(c) BDF2 scheme

Figure 1: Convergence history in time: $\mathbb{P}_{2} / \mathbb{P}_{2}$ CIP stabilized finite elements 


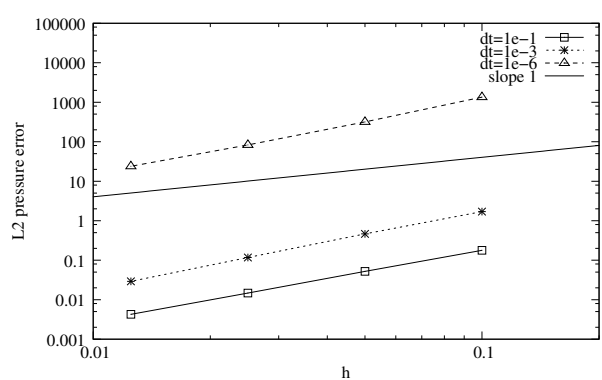

(a) Lagrange interpolant $\boldsymbol{u}_{h}^{0}=I_{h}^{1} \boldsymbol{u}_{0}$

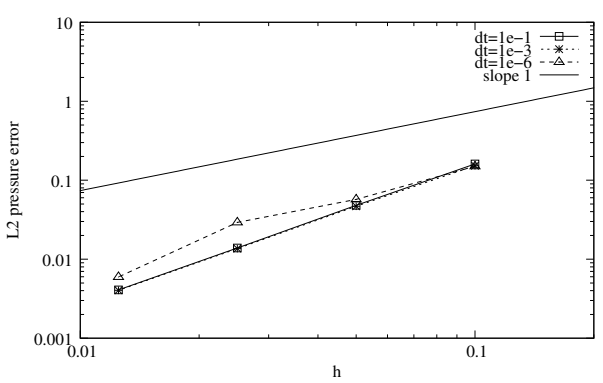

(b) Ritz-projection $\boldsymbol{u}_{h}^{0}=P_{h}^{1}\left(\boldsymbol{u}_{0}, 0\right)$

Figure 2: Convergence history: $\mathbb{P}_{1} / \mathbb{P}_{1}$ finite elements

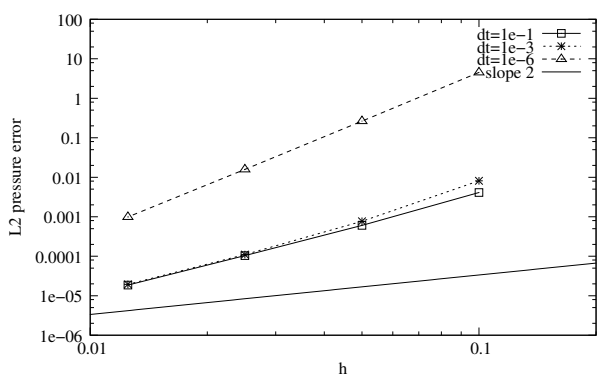

(a) Lagrange interpolant $\boldsymbol{u}_{h}^{0}=I_{h}^{2} \boldsymbol{u}_{0}$

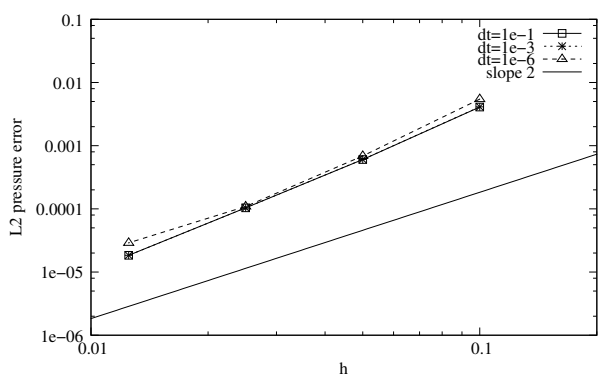

(b) Ritz-projection $\boldsymbol{u}_{h}^{0}=P_{h}^{2}\left(\boldsymbol{u}_{0}, 0\right)$

Figure 3: Convergence history: $\mathbb{P}_{2} / \mathbb{P}_{2}$ finite elements

We choose the initial data either as the Lagrange interpolant, $\boldsymbol{u}_{h}^{0}=I_{h}^{k} \boldsymbol{u}_{0}$, or as the Ritz-projection, $\boldsymbol{u}_{h}^{0}=P_{h}^{k}\left(\boldsymbol{u}_{0}, 0\right)$.

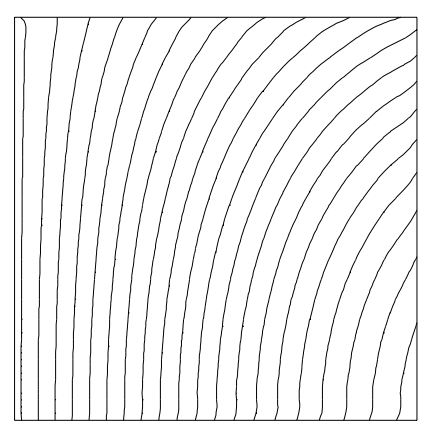

(a) $\delta t=10^{-1}$

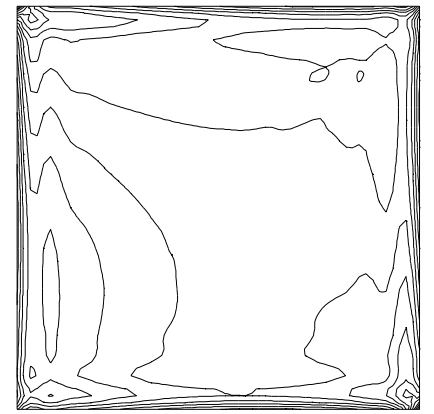

(b) $\delta t=10^{-6}$

Figure 4: Pressure contour lines with $\mathbb{P}_{2} / \mathbb{P}_{2}$ finite elements in a $40 \times 40$ mesh: $\boldsymbol{u}_{h}^{0}=I_{h}^{2} \boldsymbol{u}_{0}$

In Figure 2 we have reported the convergence history (in space) of the pressure error, at the first time step, using $\mathbb{P}_{1} / \mathbb{P}_{1}$ finite elements for different time step sizes. The pressure instability for small time steps is illustrated in Figure 


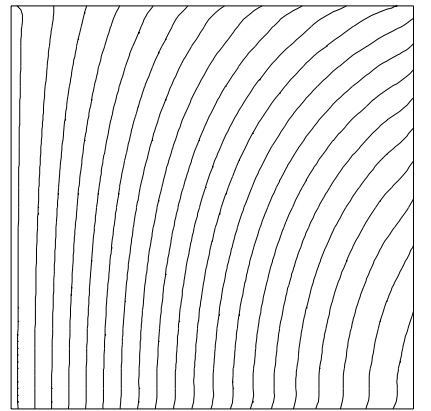

(a) $\delta t=10^{-1}$

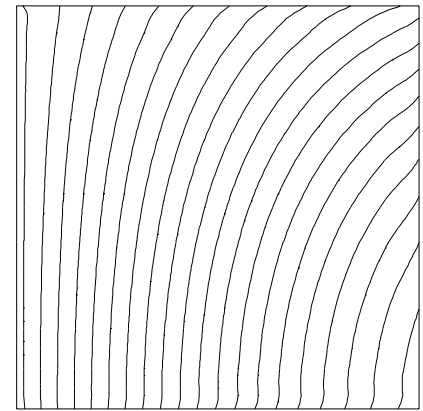

(b) $\delta t=10^{-6}$

Figure 5: Pressure contour lines with $\mathbb{P}_{2} / \mathbb{P}_{2}$ finite elements in a $40 \times 40$ mesh: $\boldsymbol{u}_{h}^{0}=P_{h}^{2}\left(\boldsymbol{u}_{0}, 0\right)$

4(a), where the initial velocity approximation is given in terms of the Lagrange interpolant. Indeed, we can observe that the pressure error has the right convergence rate in space, but it grows when the time step is decreased. On the other hand, as shown in Figure 4(b), the instability is eliminated when the initial velocity approximation is provided by the Ritz-projection, as stated in Corollary 4.3. In this case the error remains bounded (dominated by the space discretization) while reducing the time step size.

Similar results are found with $\mathbb{P}_{2} / \mathbb{P}_{2}$ finite elements, as shown in Figure 3 . In particular, we can notice, from Figures 2 (a) and 3(a), that for quadratic approximations the pressure instability shows up only for very small time steps. As a matter of fact, condition 42 is less restrictive for quadratic than for affine velocity approximations of smooth initial data. Finally, some pressure contours are reported in Figure 4 for the Lagrange interpolation, and in Figure 5 for the Ritz-projection. The pressure degradation is clearly visible in Figure 4. whereas with the Ritz-projection initialization (Figure 5) the pressure remains unconditionally stable.

\section{Conclusion}

In this paper we have proved unconditional stability and optimal error estimates, in natural norms, for pressure stabilized finite element approximations of the transient Stokes' problem. It should be noted that the extension of the present results to mixed formulations of the Poisson problem is straightforward. We have shown that for small initial time-steps the use of a pressure stabilization dependent Ritz-projection, for the initial data, is essential to avoid pressure instabilities, unless a condition between time and space discretization parameters is satisfied. From the analysis, we also conclude that a second order scheme (e.g. BDF2) can be initialized (without optimality loss) using a first step with BDF1, provided that the Ritz-projection (21) is used for the initial data.

It is interesting to note that for low order elements the weakly consistent stabilization operators still yield optimal convergence in time when used with a second order scheme. However, in case SUPG type stabilization is used for the 
convective term the convergence order in time will be lost unless full consistency is guaranteed in the stabilization term. This is why SUPG type stabilizations prompt space time finite element formulations with discontinuous approximation in time.

Somme the methods described in paragraph 3.1.1, on the other hand, may be extended to the case of the Oseen's equations, handling all Reynolds numbers, by applying the same type of stabilizing term for the convection (see [13, 6, 16, 7], for details).

\section{References}

[1] S. Badia and R. Codina. On a multiscale approach to the transient Stokes problem. Transient subscales and anisotropic space-time discretization. Preprint, 2007.

[2] R. Becker and M. Braack. A finite element pressure gradient stabilization for the Stokes equations based on local projections. Calcolo, 38(4):173-199, 2001.

[3] P. B. Bochev, M. D. Gunzburger, and R. B. Lehoucq. On stabilized finite element methods for the Stokes problem in the small time step limit. Internat. J. Numer. Methods Fluids, 53(4):573-597, 2007.

[4] D. Boffi and L. Gastaldi. Analysis of finite element approximation of evolution problems in mixed form. SIAM J. Numer. Anal., 42(4):1502-1526, 2004.

[5] M. Boman. Estimates for the $L_{2}$-projection onto continuous finite element spaces in a weighted $L_{p}$-norm. BIT, 46(2):249-260, 2006.

[6] M. Braack and E. Burman. Local projection stabilization for the Oseen problem and its interpretation as a variational multiscale method. SIAM J. Numer. Anal., 43(6):2544-2566 (electronic), 2006.

[7] M. Braack, E. Burman, V. John, and G. Lube. Stabilized finite element methods for the generalized Oseen problem. Comput. Methods Appl. Mech. Engrg., 196(4-6):853-866, 2007.

[8] J.H. Bramble, J.E. Pasciak, and O. Steinbach. On the stability of the $L^{2}$ projection in $H^{1}(\Omega)$. Math. Comp., 71(237):147-156 (electronic), 2002.

[9] F. Brezzi and M. Fortin. A minimal stabilisation procedure for mixed finite element methods. Numer. Math., 89(3):457-491, 2001.

[10] F. Brezzi and J. Pitkäranta. On the stabilization of finite element approximations of the Stokes equations. In Efficient solutions of elliptic systems (Kiel, 1984), volume 10 of Notes Numer. Fluid Mech., pages 11-19. Vieweg, Braunschweig, 1984.

[11] A.N. Brooks and T.J.R. Hughes. Streamline upwind/Petrov-Galerkin formulations for convection dominated flows with particular emphasis on the incompressible Navier-Stokes equations. Comput. Methods Appl. Mech. Engrg., 32(1-3):199-259, 1982. FENOMECH '81, Part I (Stuttgart, 1981). 
[12] E. Burman. Pressure projection stabilizations for Galerkin approximations of Stokes' and Darcy's problem. Num. Meth. Partial Differential Equations, 2007.

[13] E. Burman, M. A. Fernández, and P. Hansbo. Continuous interior penalty finite element method for Oseen's equations. SIAM J. Numer. Anal., 44(3):1248-1274 (electronic), 2006.

[14] E. Burman and P. Hansbo. Edge stabilization for the generalized Stokes problem: a continuous interior penalty method. Comput. Methods Appl. Mech. Engrg., 2005. in press.

[15] P. Clément. Approximation by finite element functions using local regularization. Rev. Française Automat. Informat. Recherche Opérationnelle Sér. Rouge Anal. Numér., 9(R-2):77-84, 1975.

[16] R. Codina. Analysis of a stabilized finite element approximation of the Oseen equations using orthogonal subscales. Appl. Numer. Math., 2007. In press.

[17] R. Codina and J. Blasco. A finite element formulation for the Stokes problem allowing equal velocity-pressure interpolation. Comput. Methods Appl. Mech. Engrg., 143(3-4):373-391, 1997.

[18] R. Codina and J. Blasco. Stabilized finite element method for the transient Navier-Stokes equations based on a pressure gradient projection. In $\mathrm{Com}$ putational mechanics (Buenos Aires, 1998), pages CD-ROM file. Centro Internac. Métodos Numér. Ing., Barcelona, 1998.

[19] R. Codina, J. Principe, O. Guasch, and S. Badia. Time dependent subscales in the stabilized finite element approximation of incompressible flow problems. Comput. Methods Appl. Mech. Engrg., 2007.

[20] M. Crouzeix and V. Thomée. The stability in $L_{p}$ and $W_{p}^{1}$ of the $L_{2}$ projection onto finite element function spaces. Math. Comp., 48(178):521$532,1987$.

[21] C. R. Dohrmann and P. B. Bochev. A stabilized finite element method for the Stokes problem based on polynomial pressure projections. Internat. J. Numer. Methods Fluids, 46(2):183-201, 2004.

[22] A. Ern and J.-L. Guermond. Theory and practice of finite elements, volume 159 of Applied Mathematical Sciences. Springer-Verlag, New York, 2004.

[23] L.P. Franca and S.L. Frey. Stabilized finite element methods. II. The incompressible Navier-Stokes equations. Comput. Methods Appl. Mech. Engrg., 99(2-3):209-233, 1992.

[24] J. Freund and R. Stenberg. On weakly imposed boundary conditions for second order problems. In M. Morandi Cecchi et al., editor, Proceedings of the Ninth Int. Conf. Finite Elements in Fluids, pages 327-336, Venice, 1995. 
[25] V. Girault and P. A. Raviart. Finite element methods for Navier-Stokes equations, volume 5 of Springer Series in Computational Mathematics. Springer-Verlag, Berlin, 1986.

[26] F. Hecht, O. Pironneau, A. Le Hyaric, and K. Ohtsuka. FreeFem $++v$. 2.11. User's Manual. LJLL, University of Paris 6.

[27] T. J. R. Hughes, L. P. Franca, and M. Balestra. A new finite element formulation for computational fluid dynamics. V. Circumventing the BabuškaBrezzi condition: a stable Petrov-Galerkin formulation of the Stokes problem accommodating equal-order interpolations. Comput. Methods Appl. Mech. Engrg., 59(1):85-99, 1986.

[28] G. Matthies, P. Skrzypacz, and L. Tobiska. A unified analysis for local projection stabilisations applied to the Oseen problem. Technical report, Otto-von-Guericke-Universität Magdeburg, 2007.

[29] J. Nitsche. Über ein Variationsprinzip zur Lösung von Dirichlet-Problemen bei Verwendung von Teilräumen, die keinen Randbedingungen unterworfen sind. Abh. Math. Sem. Univ. Hamburg, 36:9-15, 1971. Collection of articles dedicated to Lothar Collatz on his sixtieth birthday.

[30] A. Quarteroni and A. Valli. Numerical approximation of partial differential equations, volume 23 of Springer Series in Computational Mathematics. Springer-Verlag, Berlin, 1994.

[31] L. R. Scott and S. Zhang. Finite element interpolation of nonsmooth functions satisfying boundary conditions. Math. Comp., 54(190):483-493, 1990.

[32] D. Silvester. Optimal low order finite element methods for incompressible flow. Comput. Methods Appl. Mech. Engrg., 111(3-4):357-368, 1994.

[33] M. Suri. Mixed variational principles for time-dependent problems. $\mathrm{PhD}$ thesis, Carnegie-Mellon University, 1983.

[34] V. Thomée. Galerkin finite element methods for parabolic problems, volume 25 of Springer Series in Computational Mathematics. Springer-Verlag, Berlin, second edition, 2006.

\section{Contents}

\begin{tabular}{lll}
\hline & Introduction & 3
\end{tabular}

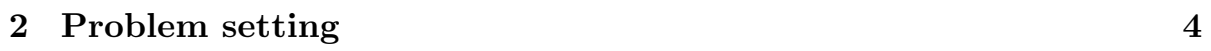

3 Space and time discretization 5

3.1 Space semi-discretization: symmetric pressure stabilized formu-

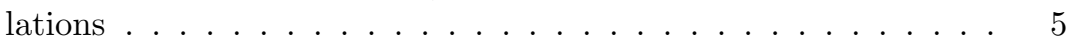

3.1 .1 Examples . . . . . . . . . . . . . . . . 7

3.1 .2 The Ritz projection operator . . . . . . . . . . . . . . . 11

$3.2 \quad$ Fully discrete formulation: time discretization . . . . . . . . . . . 13 
$\begin{array}{lll}4 & \text { Stability } & 14\end{array}$

$4.1 \quad$ First order $\mathcal{A}$-stable scheme . . . . . . . . . . . . . . . . . . . . . 14

4.2 Second order $\mathcal{A}$-stable schemes $\ldots \ldots \ldots \ldots . \ldots 18$

$\begin{array}{lll}5 & \text { Convergence } & 21\end{array}$

\begin{tabular}{|lll}
6 & Numerical experiments & 27
\end{tabular}

6.1 Convergence rate in time . . . . . . . . . . . . . . 27

6.2 Behavior in the small time step limit . . . . . . . . . . . . 28

\begin{tabular}{lll}
\hline 7 Conclusion & 31
\end{tabular} 
Unité de recherche INRIA Rocquencourt Domaine de Voluceau - Rocquencourt - BP 105 - 78153 Le Chesnay Cedex (France)

Unité de recherche INRIA Futurs : Parc Club Orsay Université - ZAC des Vignes 4, rue Jacques Monod - 91893 ORSAY Cedex (France)

Unité de recherche INRIA Lorraine : LORIA, Technopôle de Nancy-Brabois - Campus scientifique 615, rue du Jardin Botanique - BP 101 - 54602 Villers-lès-Nancy Cedex (France)

Unité de recherche INRIA Rennes : IRISA, Campus universitaire de Beaulieu - 35042 Rennes Cedex (France)

Unité de recherche INRIA Rhône-Alpes : 655, avenue de l'Europe - 38334 Montbonnot Saint-Ismier (France) Unité de recherche INRIA Sophia Antipolis : 2004, route des Lucioles - BP 93 - 06902 Sophia Antipolis Cedex (France) 